\title{
Galaxy Evolution Probe
}

\author{
Jason Glenn, ${ }^{\mathrm{a}, *}$ Charles M. Bradford, ${ }^{\mathrm{b}}$ Erik Rosolowsky $\odot,{ }^{\mathrm{c}}$ \\ Rashied Amini, ${ }^{\text {b }}$ Katherine Alatalo, ${ }^{\mathrm{d}}$ Lee Armus, ${ }^{\text {e }}$ Andrew J. Benson $\odot,{ }^{f}$

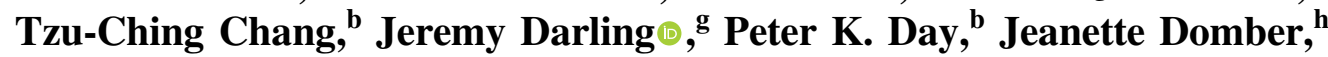 \\ Duncan Farrah $\odot,{ }^{i}$ Brandon Hensley $\odot,{ }^{j}$ Sarah Lipscy, ${ }^{\text {h }}$ Bradley Moore, ${ }^{\text {b }}$ \\ Seb Oliver $\odot,{ }^{k}$ Joanna Perido, ${ }^{\mathrm{g}}$ David Redding, ${ }^{\mathrm{b}}$ Michael Rodgers, ${ }^{\mathrm{b}}$ \\ Raphael Shirley $\odot,{ }^{\mathrm{k}}$ Howard A. Smith, ${ }^{1}$ John B. Steeves $\odot,{ }^{\mathrm{b}}$ Carole Tucker, ${ }^{\mathrm{m}}$ \\ and Jonas Zmuidzinas ${ }^{\mathrm{n}}$ \\ ${ }^{a}$ NASA Goddard Space Flight Center, Code 665, Greenbelt, Maryland, United States \\ ${ }^{\mathrm{b}}$ Jet Propulsion Lab, Pasadena, California, United States \\ ${ }^{c}$ University of Alberta, 2-115 Centennial Centre For Interdisciplinary SCS II, Edmonton, \\ Alberta, Canada \\ ${ }^{\mathrm{d}}$ Space Telescope Science Institute, Baltimore, Maryland, United States \\ ${ }^{\mathrm{e}}$ California Institute of Technology, Infrared Processing and Analysis Center, Pasadena, \\ California, United States \\ ${ }^{\mathrm{f}}$ Carnegie Observatories, Pasadena, California, United States \\ ${ }^{g}$ University of Colorado, Center for Astrophysics and Space Astronomy, Boulder, \\ Colorado, United States \\ ${ }^{\text {h} B a l l ~ A e r o s p a c e, ~ B o u l d e r, ~ C o l o r a d o, ~ U n i t e d ~ S t a t e s ~}$ \\ ${ }^{i}$ University of Hawaii Manoa, Honolulu, Hawaii, United States \\ ${ }^{j}$ Princeton University, Department of Astrophysical Sciences, Princeton, New Jersey, \\ United States \\ ${ }^{k}$ University of Sussex, Astronomy Centre, Brighton, United Kingdom \\ ${ }^{1}$ Center for Astrophysics - Harvard and Smithsonian, Cambridge, Massachusetts, \\ United States \\ ${ }^{\mathrm{m}}$ Cardiff University, School of Physics and Astronomy, Cardiff, United Kingdom \\ ${ }^{n}$ California Institute of Technology, The Division of Physics, Mathematics, and Astronomy, \\ Pasadena, California, United States
}

\begin{abstract}
The Galaxy Evolution Probe (GEP) is a concept for a mid- and far-infrared space observatory to measure key properties of large samples of galaxies with large and unbiased surveys. GEP will attempt to achieve zodiacal light and Galactic dust emission photon backgroundlimited observations by utilizing a $6-\mathrm{K}, 2.0-\mathrm{m}$ primary mirror and sensitive arrays of kinetic inductance detectors (KIDs). It will have two instrument modules: a 10 to $400 \mu \mathrm{m}$ hyperspectral imager with spectral resolution $R=\lambda / \Delta \lambda \geq 8$ (GEP-I) and a 24 to $193 \mu \mathrm{m}, R=200$ grating spectrometer (GEP-S). GEP-I surveys will identify star-forming galaxies via their thermal dust emission and simultaneously measure redshifts using polycyclic aromatic hydrocarbon emission lines. Galaxy luminosities derived from star formation and nuclear supermassive black hole accretion will be measured for each source, enabling the cosmic star formation history to be measured to much greater precision than previously possible. Using optically thin far-infrared fine-structure lines, surveys with GEP-S will measure the growth of metallicity in the hearts of galaxies over cosmic time and extraplanar gas will be mapped in spiral galaxies in the local universe to investigate feedback processes. The science case and mission architecture designed to meet the science requirements is described, and the KID and readout electronics state of the art and needed developments are described. This paper supersedes the GEP concept study report cited in it by providing new content, including: a summary of recent mid-infrared KID development, a discussion of microlens array fabrication for mid-infrared KIDs, and additional context for galaxy surveys. The reader interested in more technical details may want to consult the concept study report. (1) The Authors. Published by SPIE under a Creative Commons Attribution 4.0 Unported License. Distribution or reproduction of this work in whole or in part requires full attribution of the original publication, including its DOI. [DOI: 10.1117/1.JATIS.7.3.034004]
\end{abstract}

*Address all correspondence to Jason Glenn, jason.glenn@ nasa.gov 
Keywords: galaxy evolution; interstellar medium; mid infrared; far-infrared; kinetic inductance detectors; probe class.

Paper 20152 received Oct. 9, 2020; accepted for publication Jul. 27, 2021; published online Aug. 26, 2021.

\section{Introduction}

\subsection{Galaxy Evolution Probe in Context}

Tracing the mass assembly history of galaxies is an essential component of understanding the origins of the Hubble sequence and of using galaxies as cosmological probes of dark matter and dark energy. Observational and theoretical work over the past three decades have established a baseline framework for galaxy assembly in a cosmological context. These studies have shown that stellar and black hole mass assembly varies substantially with redshift, increasing by more than an order of magnitude between the local universe and $z=2,{ }^{1-3}$ though the behavior at higher redshifts is uncertain. ${ }^{4}$ Environment and large-scale structure profoundly affect galaxy assembly, with factors such as local galaxy density and dark matter halo properties known to play key roles in shaping the galaxy mass function.$^{5-8}$ Finally, there is a complex and subtle relationship between star formation and active galactic nucleus (AGN) activity, ${ }^{9}$ including both positive and negative feedback effects. ${ }^{10-13}$ Infrared surveys from facilities including IRAS, ${ }^{14}$ ISO,${ }^{15}$ AKARI,${ }^{16}$ Herschel,${ }^{17}$ WISE,${ }^{18}$ and Spitzer ${ }^{19}$ have played fundamental roles in these studies, since a substantial fraction of all galaxies over the history of the universe were permeated with dust through their most active evolutionary stages.

Building upon decades of observational and theoretical work, collectively a data-driven, selfconsistent model for galaxy evolution that starts from cosmology, incorporates stellar dynamics and evolution, and includes interstellar processes, star formation, and supermassive black hole growth is within reach. Achieving this goal requires addressing the questions that have arisen from previous and current generations of multiwavelength surveys. These include: what was the role of feedback from black holes and stars themselves in regulating star-formation? How did galaxies' external environments and internal contents influence their evolutionary trajectories? When were the Universe's heavy elements formed by stars in galaxies and how did they escape into the circumgalactic and intergalactic medium?

Answering these questions will require large panchromatic surveys measuring bulk properties of hundreds of thousands to millions of galaxies over most of cosmic history paired with detailed high angular and spectral resolution studies of gas and star formation in individual galaxies. Such large samples are needed to precisely disentangle the effects of redshift and environment in driving galaxy assembly. High-resolution observations of representative and outlier galaxies identified in surveys trace detailed physical processes on a galaxy-by-galaxy basis, enabling astrophysical processes to be mapped onto cosmological processes.

New mid- and far-infrared observations enabled by the rapid advances in infrared detectors and technolog $y^{20}$ are a vital component of these next-generation surveys. They require sensitivity to detect Milky Way-type galaxies at $z=2\left(L^{*} \sim 10^{12} L_{\odot}\right)$, prior to when most stellar mass had been assembled. Star formation rates and supermassive black hole accretion rates should be measured over a full range of cosmic environments, from isolated field galaxies, to galaxies in groups and in massive clusters. Measuring the heating of dust and gas by star formation and AGN is needed to understand how stellar and supermassive black hole growth were linked over cosmic time. Spectral mapping capability is needed for unbiased spatial-spectral surveys, line luminosities and intensity mapping, and line mapping of nearby galaxies to measure gas column densities, ionization parameters, and metallicities with tracers unaffected by dust obscuration. Crucially, galaxies detected by their dust continuum emission must have measured redshifts so their epochs and luminosity distances are known and their multiwavelength counterparts can be identified.

The Galaxy Evolution Probe (GEP) is a NASA Astrophysics Probe concept that capitalizes on new detector capability to address these questions with a powerful mid- and far-infrared toolset. GEP will measure star-formation rates and detect AGN even under conditions of heavy dust 
extinction. It will measure supermassive black hole accretion rates to address the connection between the masses of stellar populations and supermassive black holes. The same observations will measure metallicities with extinction-free tracers to observe growth of metals over the last two-third of the Universe's age. In nearby galaxies, GEP will observe feedback between starformation, AGN, and the interstellar medium to understand the processes that regulate starformation. Mapping nearby galaxies and the Galactic interstellar medium will reveal the energy balance by measuring the total interstellar material mass, ionization state, and the local radiation field using fine-structure transitions of ions, polycyclic aromatic hydrocarbon (PAH) molecules, and the mid-infrared dust continuum.

\subsection{GEP Concept}

GEP (Fig. 1) is designed with a 2.0-m primary mirror that will be cooled to $6 \mathrm{~K}$ to enable sensitivity limited by photon shot noise from foreground astrophysical sources: zodiacal dust emission and Galactic dust emission. There is one scientific instrument with two modules: an imager, GEP-I, and a dispersive spectrometer, GEP-S. GEP-I has 23 photometric bands distributed on the focal plane: 18 resolution $R=\lambda / \Delta \lambda=8$ bands from 10 to $95 \mu$ m designed to measure redshifts with PAHs and mid-infrared spectral energy distributions, and five resolution $R=3.5$ bands from 95 to $400 \mu \mathrm{m}$ to measure dust spectral energy distributions encompassing the peak to beyond $z=2$. GEP-S utilizes four long-slit grating spectrometers with spectral resolution $R=$ 200 from 24 to $193 \mu \mathrm{m}$. Both modules baseline arrays of kinetic inductance detectors (KIDs) cooled to $100 \mathrm{mK}$ by a multistage adiabatic demagnetization refrigerator (ADR) backed by a hybrid Joule-Thomson and Stirling cryocooler, which will also cool the telescope and coupling optics. GEP launch is targeted for January 1, 2029, with a planned mission duration of 4 years at Earth-Sun L2 (Table 1).

GEP is optimized for large, multitiered surveys for galaxies detected by their mid- and farinfrared emission from dust, PAHs, and atomic fine-structure lines. The GEP reference mission includes two types of surveys: photometric hyperspectral surveys with GEP-I and spectroscopic surveys with GEP-S. The GEP-I survey areas will be 3,30, and 300 square degrees and an all-sky survey (Fig. 2). The spectral surveys with $<1 \%$-level redshift precision will cover a range of lowand high-ionization atomic fine-structure lines. Spectral surveys will consist of "blind" surveys

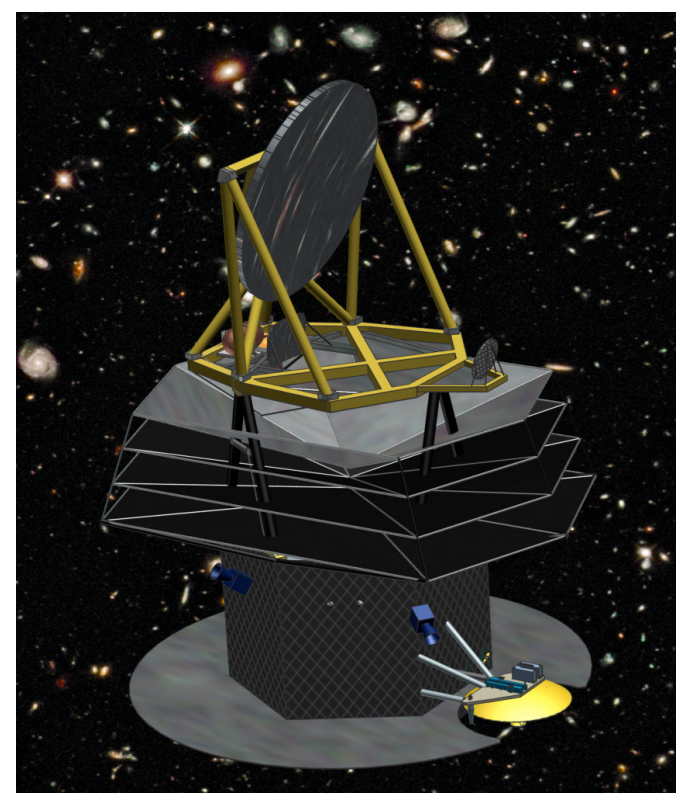

Fig. 1 Engineering rendering of GEP. From top to bottom, the prominent visible structures are the 2.0-m primary mirror and support structure, the sunshields, the bus with radiative panels, the sunward-facing solar-panel skirt, and the high-gain antenna. Figure reprinted from Fig. 3 of Ref. 23, with permission. 
Table 1 Basic GEP parameters.

\begin{tabular}{ll}
\hline \hline Parameter & \multicolumn{1}{c}{ Quantity } \\
\hline Target launch date & January 1,2029 \\
Orbit & Sun-Earth L2 \\
Observing mode & Dedicated surveys \\
Mission duration & 4 years \\
Telescope & $2.0 \mathrm{~m}, 4 \mathrm{~K}$, unobscured, Au-coated SiC \\
GEP-I wavebands & 23 bands covering 10 to $400 \mu \mathrm{m}$ \\
GEP-I $R(\lambda / \Delta \lambda)$ & 8 (10 to $95 \mu \mathrm{m}), 3.5$ (95 to $400 \mu \mathrm{m})$ \\
GEP-I surveys and target depths (obtainable & All sky, $\sim 1 \mathrm{mJy}$ \\
with photon background-limited sensitivities) & 300 sq. deg. $\sim 50 \mu \mathrm{Jy}$ \\
& 30 sq. deg. $\sim 20 \mu \mathrm{Jy}$ \\
& 3 sq. deg. $\sim 5 \mu \mathrm{Jy}$ \\
GEP-S bands & 24 to 42,40 to 70,66 to 116,110 to $193 \mu \mathrm{m}$ \\
GEP-S $R(\lambda / \Delta \lambda)$ & 200 \\
\hline \hline
\end{tabular}

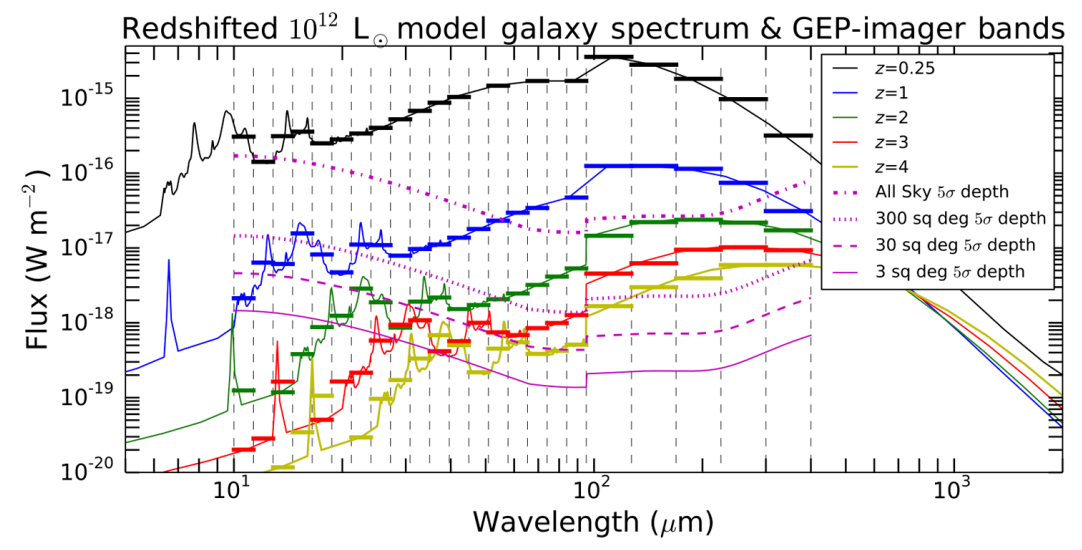

Fig. 2 GEP will detect $10^{12} L_{\odot}$ galaxies at $z=2$ and will measure redshifts with PAHs to at least $z=4$ for bright galaxies and $z \approx 7$ for gravitationally lensed galaxies. The spectra display $\mathrm{PAH}$ emission lines, silicate absorption at $10 \mu \mathrm{m}$ (in the rest frame), a rising mid-infrared continuum from warm dust, and a peak just longward of $100 \mu \mathrm{m}$ from cold dust. The spectra are binned into GEP-I's wavebands, whose edges are demarcated by dashed vertical lines. The bandwidths change abruptly at $95 \mu \mathrm{m}$ from $R=8$ to $R=3.5$ because the broad PAH emission lines are not expected to be bright enough for redshift determination for large numbers of galaxies. Atomic finestructure emission lines and molecular lines are not shown. Magenta lines indicate $5 \sigma$ survey depths assuming photon background-limited sensitivities.

utilizing a long-slit configuration, intensity mapping, and follow-up, deep pointed observations of galaxies identified in the GEP-I surveys, and regions of the Milky Way and nearby galaxies. GEP will require 4 years to do these surveys; however, it utilizes no expendable cryogens, and a Guest Observer mission can be envisioned after the surveys are completed.

GEP shares common elements with two other cryogenic far-infrared space missions under study: SPICA, an ESA-JAXA collaboration in a similar cost class to GEP, and Origins Space 
Telescope (OST), a NASA concept study for a future flagship infrared mission. SPICA's design features a $2.5-\mathrm{m}, 6-$ to $8-\mathrm{K}$ telescope and a spectrometer using sensitive bolometer arrays, although it does not have the 23-band, moderate spectral resolution hyperspectral imaging that is central to GEP's architecture. OST is designed with a 5.9-m, 4.5-K telescope; its earliest possible realization is well into the 2030 decade.

\subsection{This Paper}

GEP was described in the concept study report submitted to NASA, ${ }^{21}$ and its science case was briefly summarized in a short white paper submitted to the 2020 Astronomy Decadal Survey. ${ }^{22}$ The initial design concept for the mission and the optical design were described by Glenn et al., ${ }^{23}$ and the cryogenic design was described by Moore et al. ${ }^{24}$ This paper summarizes the design and science and provides new content: new engineering design, a summary of recent mid-infrared KID development, a discussion of microlens array fabrication for mid-infrared KIDs, and additional context for galaxy surveys.

\section{Science Goals}

GEP has two overarching science goals. The first is to map the history of galaxy growth through star formation and accretion by supermassive black holes and to characterize the relationship between these processes. The second is to measure the growth of metals in galaxies and the changing of star-formation interstellar medium environments in galaxies over cosmic time. These science goals are broken down into specific objectives, which are translated into requirements that drive the GEP design in the concept study science traceability matrix. ${ }^{21}$

Broadly, the star formation began in the first billion years of the Universe, rose to a peak or broad plateau around $z=2-3$, and then declined sharply. ${ }^{2}$ Despite substantial success over the past decades in understanding the average star formation and supermassive black hole accretion rates with redshift, large uncertainties and significant questions about galaxy evolution still exist, including: star formation rates derived from infrared galaxy surveys of limited size have not probed low luminosities ${ }^{25}$ (essentially, below $L^{*}$ ) and are limited by sample variance, redshifts of large samples of far-infrared continuum-detected galaxies are uncertain, and extinction correction in rest-frame ultraviolet observations is substantial and sometimes uncertain. ${ }^{2}$ GEP is designed to address these concerns with large, deep infrared surveys for star-forming galaxies to meet its first objective: to measure the coevolution of star formation and supermassive black hole growth in galaxies. GEP-I will yield star formation rates from far-infrared luminosities ${ }^{26,27}$ and separate AGN and star formation contributions to total infrared luminosities via mid-infrared spectral shapes,$^{28}$ even in cases of heavy extinction because the dust emission is almost always optically thin. The 23-band GEP-I photometry of the mid-infrared PAH features will yield redshifts for the galaxies. PAHs have been detected spectroscopically with Spitzer at high redshifts, e.g., $z=1.09$ and $2.96^{29}$ and $z=4.055,{ }^{30}$ in galaxies with and without prominent AGN. From mid- and far-infrared fine-structure lines, GEP-S will measure star formation rates ${ }^{31,32}$ and supermassive black hole accretion rates. ${ }^{32}$

GEP will access the early epochs of galaxy growth $(z>3)$ by utilizing the brightening from gravitational lensing. It is clear that much of the star formation and black hole growth in massive galaxies since reionization occurred in dusty regions, and there is considerable uncertainty about how much star formation rate density may be missed in the ultraviolet census of that early phase of galaxy evolution. ${ }^{2}$ Wide-area GEP-I surveys taking advantage of the brightening provided by gravitational lensing will address this problem with $10^{4}$ lensed high- $z$ galaxies.

Understanding the role of accreting supermassive black holes in galaxy evolution requires infrared observations because these processes occur on small scales at the centers of galaxies, in most cases obscured by the very gas and dust that is accreting. A principal objective of GEP is to identify obscured AGN in galaxies and relate their accretion luminosities to their star formation rates. GEP will identify and quantify luminosities of dust-obscured AGN via mid-infrared spectral signatures, including the continuum shape with GEP-I and high-ionization fine-structure atomic transitions with GEP-S, such as [Ne V] at rest-frame wavelengths of 14.3 and $24.3 \mu \mathrm{m}$

J. Astron. Telesc. Instrum. Syst. $\quad$ 034004-5 Jul-Sep 2021 • Vol. 7(3) 
and [O IV] at $25.9 \mu \mathrm{m}$. GEP complements x-ray detection of AGN because x-ray observations can miss Compton-thick AGN or underestimate accretion rates.

The masses of supermassive black holes in the centers of modern-day galaxies are well correlated with galaxies' bulge masses. ${ }^{33,34}$ This has led to the now commonly adopted hypothesis that a feedback loop exists in which AGN activity governs the rate of star-formation in massive galaxies or at least in galactic bulges. ${ }^{35}$ Theoretical models ${ }^{10,36-38}$ invoke AGN feedback as a primary mechanism to explain the observed distribution of galaxy masses today. Without AGN feedback, models are unable to explain the low ratio of galactic stellar mass to halo mass for high-mass galaxies. ${ }^{39}$ However, the efficiency of AGN feedback for regulating star formation has not been established empirically and remains controversial. ${ }^{12,40}$ GEP's goal is to determine how feedback from buried accreting black holes was related to the decline of star-formation. GEP will assess the role of feedback in galaxies by searching for faint wings or asymmetries in the velocity profiles of bright fine-structure lines in stacked spectra of AGN and starburst samples. Such linewing signatures have been measured interferometrically in CO (e.g., Ref. 13) as well as in the optical (e.g., Ref. 41). They can have velocities in excess of $1000 \mathrm{~km} / \mathrm{s}$, particularly for the AGN sources. Given the modest spectral resolving power of GEP-S ( $R=200$ or $1500 \mathrm{~km} / \mathrm{s})$, GEP's far-IR measurement will be accomplished with a combination of (a) high signal-to-noise ratio ( $\sim 100$ or greater) in the main line core for either the high-redshift spectral stacks or individual nearby galaxy spectra and (b) knowledge of the spectral response function of the instrument, with fidelity to 1 part in 500 or better. The excellent raw sensitivity of GEP-S, combined with the fact that we are considering the brightest lines in the spectrum, will satisfy the first condition. For the second, a combination of laboratory measurements preflight and measurements of narrow Milky Way line-emitting standards should provide ample opportunity for accurate spectral calibration. In nearby galaxies, GEP will directly obtain a spatially resolved view of feedback and its effects with a spectroscopic study of galactic outflows and fountains in local galaxies in various atomic fine-structure emission lines (e.g., [C II], [N II], and [O III]).

GEP is designed not only to measure the star formation rates of galaxies over cosmic time but also the evolving conditions of star formation regions. Specifically, the measurements of finestructure lines, including [C II], [N II], and [O III], will be used to infer the mass and density of the interstellar medium gas and the hardness of the ambient radiation, which has implications for the stellar initial mass function, and the density of $\mathrm{H}$ II regions from which the interstellar pressure can be inferred. The resulting relations between metallicity, star-formation rate, and other galaxy properties will inform models of galactic winds by placing constraints on the presence of gas-phase metals at their source.

Metallicity represents the integrated effects of star-formation, inflow, and outflow from galaxies. A key objective of GEP is to track the buildup of heavy elements in galaxies over the peak epoch of star-formation utilizing spectroscopic surveys of metals in atomic gas (and secondarily lower-resolution spectra of PAHs and dust). Metallicities of galaxies have not been measured beyond the local universe with the extinction-free probes afforded by far-infrared atomic finestructure lines. GEP will measure the metallicity in galaxies down to $L^{*}=10^{12} L_{\odot}$ galaxies at $z=2$ using the nitrogen-to-oxygen ratio measured with [OIII] and [NIII] fine-structure lines. This ratio measures metallicity through its connection to stellar processing since nitrogen is a secondary nucleosynthesis product and comes on later in the nucleosynthesis process. The nitrogen/oxygen approach has been used with optical lines but the far-IR lines offer the dual benefit of dust immunity and temperature insensitivity. ${ }^{42}$ Alternatively, for the highest redshifts, metallicities will be measured using a diagnostic formed from the mid-IR tracers of neon and sulfur. This has been shown to track metallicity in photoionization models and has been validated in a careful study of local galaxies that have Spitzer mid-IR spectroscopy in hand. ${ }^{43}$

\section{Mission Architecture: Thermal Design and Observing Modes}

GEP is designed as a Class B mission. A NASA Class B mission is a mission whose loss would be highly impactful to national science objectives and therefore that only low risks to mission success will be tolerated. Less risk is tolerated for Class A missions and more for Classes C and D. GEP is based on the Ball Aerospace BCP2000 reference bus, of Kepler heritage, customized 
to meet the science and mission requirements of GEP. The 4-year survey program is divided into a GEP-I campaign and a GEP-S campaign, from which $350 \mathrm{~TB}$ of observational data will be downlinked. Mission details can be found in the concept study report. ${ }^{21}$

GEP's thermal system employs multiple passive and active stages to meet the temperature intercept requirements of the instruments and optical assembly (Fig. 3). A continuous multistage ADR provides cooling for the detectors at $100 \mathrm{mK}$, with a 1-K thermal intercept to reduce thermal noise and parasitic loads. A hybrid Joule-Thomson/Stirling cryocooler intercepts heat at $4 \mathrm{~K}$ from the ADR and from the cryogenic amplifiers and parasitic loads. The hybrid cooler has an $18 \mathrm{~K}$ intercept to cool a second stage of amplifiers for the detector signals and an active shield.

The sunshield assembly consists of three passively cooled reflective shields with a total area of $33 \mathrm{~m}^{2}$ and a $9.77-\mathrm{m}^{2}$ active shield located above the sunshield under the focal plane boxes. The substrate of all four shields is an internally self-supporting 1-mm-thick M55J laminate with an areal density of $29.5 \mathrm{~kg} \mathrm{~m}^{-2}$. The reflective coating on the three warmer, passively cooled sunshields consists of a thin ( 0.005 in.) layer of aluminized Kapton adhered to the surface. The bottom surface of the actively cooled sunshield is also aluminized Kapton; however, the top surface is a 1-mm thick, high purity aluminum thermal spreader layer. Fiberglass composite struts provide intershield supports at the perimeter to accept launch loads. The passively cooled shields intercept conducted loads from the bipods and harnesses and radiative loads from the Sun. With these nondeployable sunshields, the spacecraft can tilt $\pm 21.6 \mathrm{deg}$, maintaining all cryogenic components in the shadow cone and thus preserve thermal system operability. The cooler and ADR electronics dissipate heat at ambient temperature $(\sim 300 \mathrm{~K})$, along with

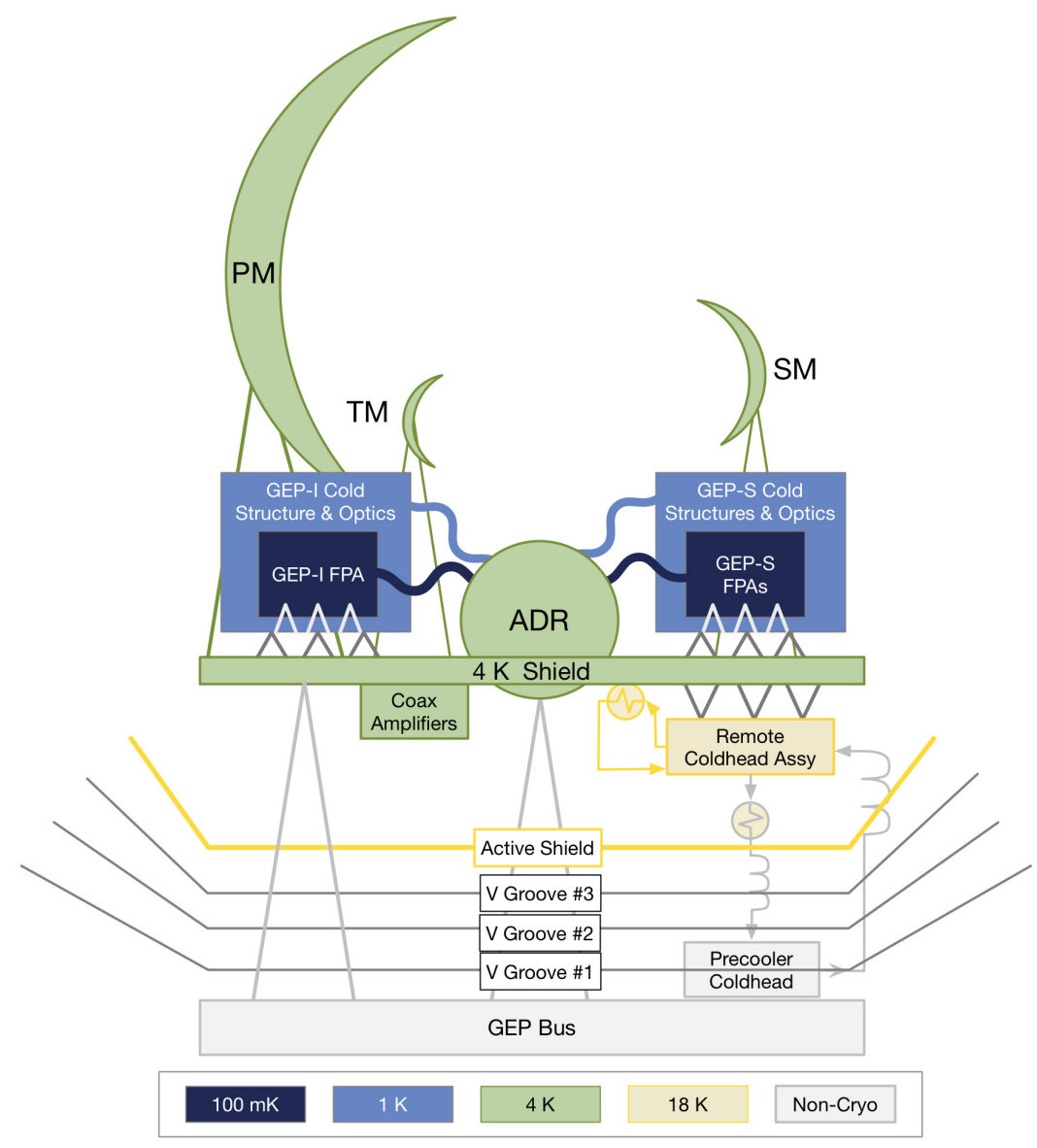

Fig. 3 GEP thermal configuration. GEPs thermal design uses active and passive cooling to maintain $100 \mathrm{mK}$ required by the focal planes, $6 \mathrm{~K}$ for the optical assembly, including the telescope mirrors, and $18 \mathrm{~K}$ for the cryogenic amplifiers. This configuration supersedes the configuration in Ref. 24. PM, primary mirror; SM, secondary mirror; TM, tertiary mirror; FPA, focal plane array. 
all bus components, which is radiated by $10.5 \mathrm{~m}^{2}$ of radiators mounted on the bus behind the solar panels.

The GEP-I and GEP-S instrument modules will observe one at a time and use the same readout electronics. They share a scan survey observing mode, where mapping is performed as the spacecraft slews at $\sim 60^{\prime \prime} \mathrm{s}^{-1}$. The scan survey mode will be used for all the GEP-I surveys, for GEP-S's 1.5 and 100 square degree surveys, and for mapping of nearby galaxies. GEP-S will also have a pointed observation mode in which a chopping mirror, with a total throw of $\pm 0.2 \mathrm{deg}$, modulates the signal for $1 / f$ noise mitigation. One 5-h DSN pass per day will be required to downlink $0.24 \mathrm{~TB}$, during which the all-sky survey will be conducted by spinning the spacecraft while the high-gain antenna is pointed at Earth. The intent is to do the entire all-sky survey in this mode while spinning at the same rate as the data taken in the nominal scan mode. If it is determined that the scan rate must be different for the all-sky mode than for nominal scanning, a separate calibration will be required.

\section{Science Instrument}

The GEP payload includes the optical telescope assembly, the GEP instrument comprised of a hyperspectral imager module (GEP-I) and four spectrometer modules (GEP-S), and a payload thermal subsystem (Fig. 4).

\subsection{Optical Design}

The fundamental optical design requirement is to collect light with a 2.0-m diameter primary mirror and to form an $f / 9$ focus. An unobscured three-mirror astigmat (TMA) is an oft-used configuration that is well suited to the first-order optical and mechanical requirements of this system. The powered mirrors are all conic shapes with parent surfaces that have mutual tilts and decentrations to reduce wavefront error. The baseline for the GEP primary, secondary, and tertiary and chopping mirrors is unactuated silicon carbide $(\mathrm{SiC})$, as was flown on the Herschel Space Observatory and Gaia. GEP's optical design and simulated performance are described in detail in Ref. 23.

The five instrument interface planes are nearly coplanar at the common focal surface of the TMA, with minor differences in final focus to minimize wavefront error in each channel. Based on the sizes of the focal-plane array and the spectrometer slits and enclosures, the optimized field of view is $0.81 \mathrm{deg} \times 0.88 \mathrm{deg}$, in excess of what is required for the instrument modules. The centers of the GEP-S slits are in the plane of symmetry of the TMA so that they can be untilted

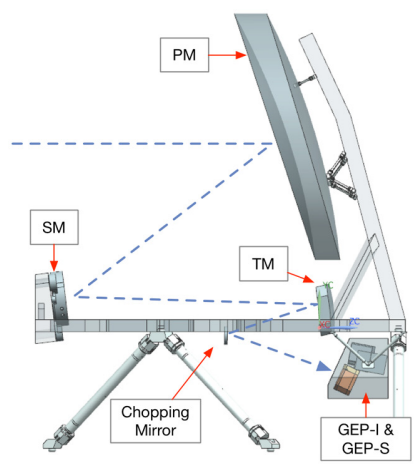

(a)

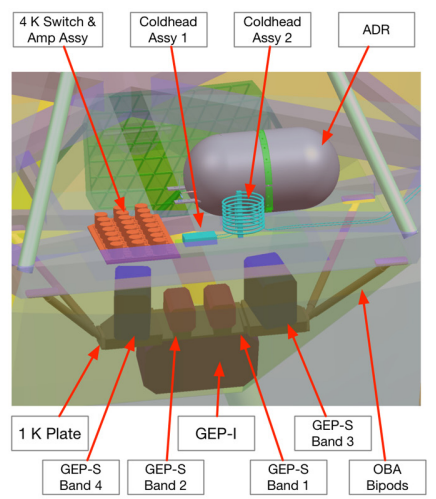

(b)

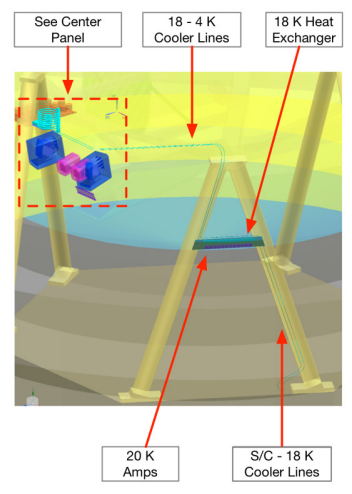

(c)

Fig. 4 (a) Side view of the GEP optical configuration showing its TMA design. A field stop is located between the secondary and tertiary mirrors. The fourth optic is a chopping mirror located at an image of the primary mirror, forming a pupil stop. The GEP-I and GEP-S modules are in the lower right. (b) Detail of GEP-I placement and the four GEP-S focal planes along with the ADR and $4 \mathrm{~K}$ cryogenic assembly. Reprinted from Fig. 11 of Ref. 23, with modifications and permission. (c) Detail of the $18 \mathrm{~K}$ assembly located near the focal plane assembly. 
with respect to the central ray to each spectrometer. Stray light suppression is achieved with a pupil stop and a field stop.

To meet the extragalactic confusion requirement (Sec. 5.3), the primary mirror is required to be diffraction limited at $\lambda=24 \mu \mathrm{m}$. However, the root-mean-square (RMS) wavefront error across the field of view in the optical design is diffraction limited at the minimum wavelength of $10 \mu \mathrm{m}$. The $100 \%$ encircled energy diameter is $\leq 1.45^{\prime \prime}$, much less than the $3.43^{\prime \prime}$ detector pixel size (see Sec. 4.2).

\subsection{GEP-I Hyperspectral Imager}

GEP-I is designed to obtain repeated measurements in each of the 23 wavebands to build survey depth by continuously scanning areas of sky covered by each survey. Each waveband occupies the same amount of focal plane area, 0.002 square degrees, half of which is occupied by detectors and half of which is allotted for bandpass filter mounting in the current design configuration (Figs. 5). Bands 1 to 18 have spectral resolution $R=\lambda / \Delta \lambda=8$, whereas bands 19 to 23 are $R=3.5$. It is likely that the mid-infrared (e.g., 10 to $26 \mu \mathrm{m}$ ) bandpass filters could be replaced with linear-variable filters, resulting a spectral resolution of at least $R=20$, which will be the subject of a future investigation.

GEP-I will have 25,735 KIDs. GEP I's optical design performance is diffraction limited at $10 \mu \mathrm{m}$; however, the primary mirror is specified to be diffraction limited at $24 \mu \mathrm{m}$, corresponding to $3^{\prime \prime}$ (FWHM) beam size. The primary reason for this is that the smallest KID pixel size we expect to be able to fabricate without exceeding the readout bandwidth is $300 \mu \mathrm{m} \times 300 \mu \mathrm{m}$ (the number of detectors is inversely proportional to their physical area), which corresponds to $3.43^{\prime \prime}$. With 3" beams and 3.43" pixels, the full angular resolution of the telescope will not be taken advantage of for GEP-I bands 1 to 9 (up to $29 \mu \mathrm{m}$ ), although all flux density will be recovered. For bands 9 to 23, the pixel size will be less than the beam FWHM. Should greater bandwidth become feasible through improvements in data acquisition and computing speed (a very likely development), the pixel sizes can be reduced to less than the beam FWHM.

\subsection{GEP-S Long-Slit Spectrometer}

GEP's spectrometer was designed to meet the science requirements calling for observing midand far-infrared atomic fine-structure lines from galaxies over a range of redshifts. Specifically, the $24.3-\mu \mathrm{m}[\mathrm{Ne} \mathrm{V}]$ line starting at $z=0$ for AGN identification and the $63.2 \mu \mathrm{m}[\mathrm{O} \mathrm{I}]$ line at $z=2$ for probing star-forming galaxies at or beyond the cosmic peak. The entire bandwidth should be available to identify spectral lines for galaxies of unknown redshift. Sufficient spectral resolution is required to achieve good sensitivity through dispersion of the astrophysical background photons. Spectral resolution $R=200$ meets these requirements.

GEP-S's design is implemented with four diffraction gratings, each with fractional bandwidth of 1.6 , identified as bands 1 to 4 (Table 1), with a total of 24,640 KIDs. The ray trace

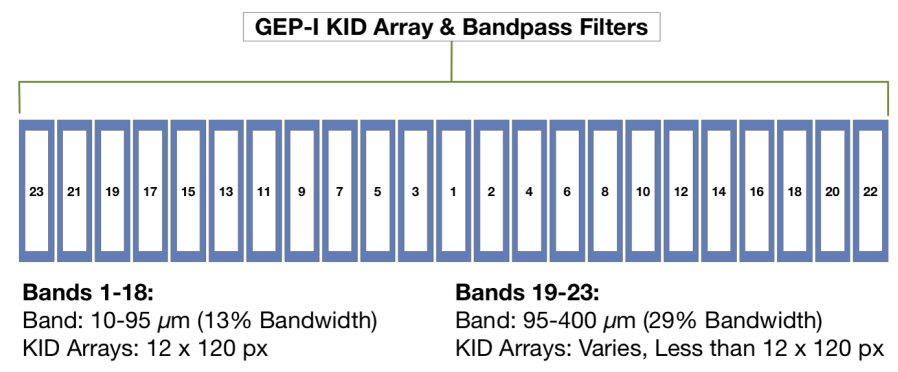

Fig. 5 GEP-I focal plane layout configuration. While the entire focal plane shown is diffraction limited at $\lambda=10 \mu \mathrm{m}$, the shortest wavebands are located at the center where the optical quality is the highest. Bands $1(10.0$ to $11.3 \mu \mathrm{m})$ through 15 (57.7 to $65.4 \mu \mathrm{m}$ ) have $1440 \mathrm{KIDs}$ (arrays of $12 \times 120$ ), with $3.43^{\prime \prime}$ pixels (300 $\mu \mathrm{m}$ square). The longer-wavelength bands have fewer, larger KIDs such that the focal plane area occupied by each band is approximately the same. For example, band 23 (300 to $400 \mu \mathrm{m}$ ) has $46 \mathrm{KIDs} 1560 \mu \mathrm{m}$ square. 
(a)

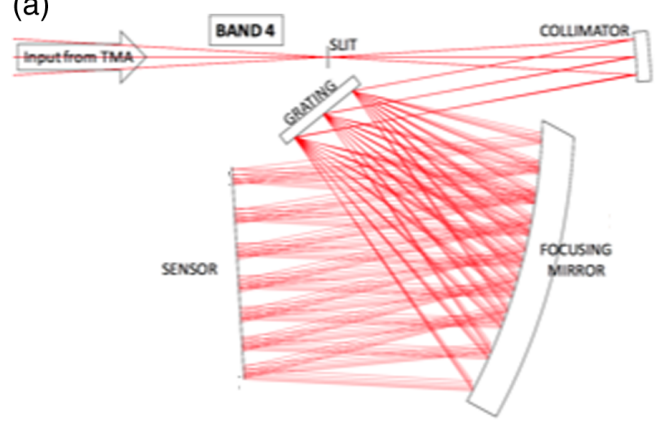

F984-SPECr-rev'

(b)

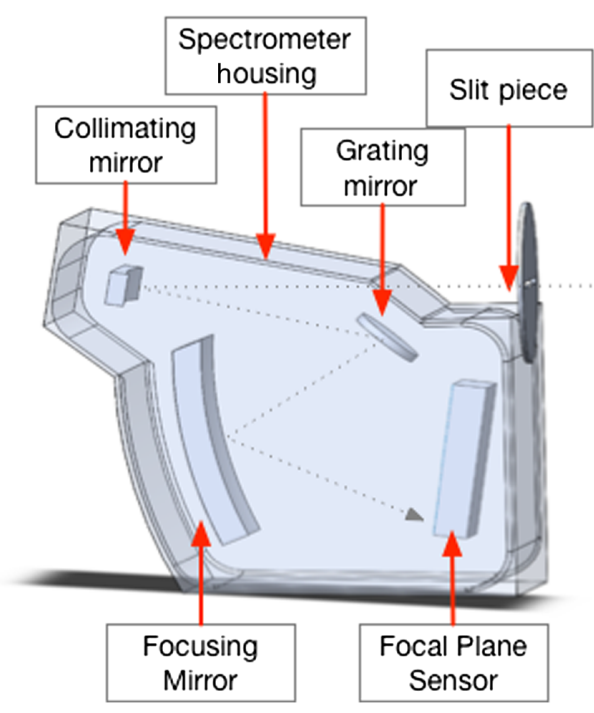

Fig. 6 (a) Ray-trace of GEP-S's band 4 spectrometer module. Reprinted from Fig. 6 of Ref. 23, with permission. (b) GEP-S band 4 mechanical layout viewed mirror imaged left-to-right with respect to the top figure.

diagram and CAD model of band 4 is shown in Fig. 6. Each band, or diffraction grating module, is comprised of an enclosure, a slit, a collimator, a grating operated in first order, a focusing mirror, and an array of KIDs. The long slit lengths enable spectral mapping. As with GEPI, the shortest-wavelength bands 1 and 2 are placed nearest to the center of the field of view where the optical performance is the best. A chopping mirror [Fig. 4(a)] is included for detector modulation for staring GEP-S observations.

\subsection{Kinetic Inductance Detectors and Readout Electronics}

KIDs have been baselined for the GEP-I and GEP-S focal plane arrays. KIDs are high-quality factor superconducting resonators whose resonances vary with absorbed light through modulation of kinetic inductance by pair breaking. ${ }^{44}$ They are in wide use in recent and imminent ground-based and suborbital optical, far-infrared, submillimeter, and millimeter-wave instruments (Fig. 7).

Two other detector array technologies were considered for GEP: transition-edge sensors (TESs) and Si:As IBCs (impurity band conductors). KIDs are not as technologically mature as TESs but their simple fabrication and focal plane electronics make them advantageous, partially because TESs require complex hybridization with SQUID readouts. Like KIDs, TESs have not been demonstrated in the 10 to $15 \mu \mathrm{m}$ wavelength regime. Si:As IBCs are technologically mature but they are unusable beyond $28 \mu \mathrm{m}$, so KIDs (or TESs) would still be needed for 28 to $400 \mu \mathrm{m}$. However, pixel pitches and operating temperature requirements for IBCs (7 K) and KIDs $(100 \mathrm{mK})$ are very different, and the divergent optical and cryogenic requirements 


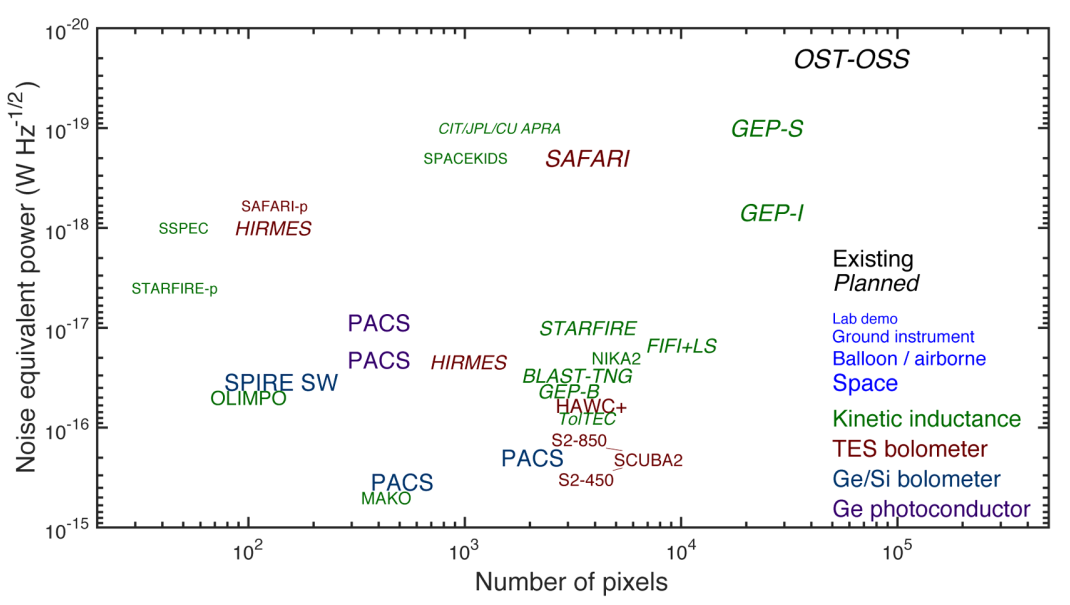

Fig. 7 Far-IR detector technology has made considerable progress toward larger, more sensitive arrays over the past decade. Detector sensitivity is shown versus numbers of detectors for existing (normal font) and planned (italic font) instrumentation. The text colors indicate the type of detector technology and the font sizes are used to differentiate the testing environment or intended application of the technology. GEP-I sensitivity requirements have been met by SPACEKIDs, ${ }^{45}$ whereas GEP-S will require further sensitivity improvement for background-limited observations. Both GEP-I and GEP-S require KIDs at mid-infrared wavelengths, for which development efforts are underway. ${ }^{46}$ Others plotted are BLAST-TNG, ${ }^{47}$ FIFI+LS (proposed), HIRMES, ${ }^{48}$ MAKO, ${ }^{49,50}$ NIKA2, ${ }^{51}$ OLIMPO, ${ }^{52}$ OST-OSS, ${ }^{53}$ PACS, ${ }^{54}$ SAFARI-p, ${ }^{55}$ SAFARI, ${ }^{56}$ SCUBA2, ${ }^{57}$ SPIRE, ${ }^{58}$ SuperSpec, ${ }^{59}$ STARFIRE-p, ${ }^{46,60}$ STARFIRE, ${ }^{61}$ and ToITEC. ${ }^{62}$ In addition to its own scientific program, GEP fills an important role between the detector technology achievable in the next few years and possible future flagships such as OST.

would effectively necessitate two instruments each for GEP-I and GEP-S, which would drive up complexity, cost, and risk. Thus, KIDs have been adopted as the baseline detector technology for all of the wavebands of GEP-I and GEP-S.

GEP-I and GEP-S will utilize backilluminated, lumped-element, microlens-coupled aluminum KIDs. Each KID is a superconducting thin-film microresonator, comprised of an absorbing meandered inductor and an interdigitated capacitor deposited on a silicon substrate. Light is concentrated onto the inductors by a microlens array. The geometry of the inductor controls the absorption characteristics as a function of wavelength and polarization. Absorption of photons changes the kinetic inductance, producing a small shift in the resonant frequency and resonance depth, which are detected in the transmitted probe signal. To enable frequency-multiplexed readout with a minimal bandwidth requirement, the interdigitated capacitors are unique for each pixel, yielding different resonant frequencies separated by several resonance line widths. Submillimeter-wavelength KIDs with sensitivity sufficient for GEP-I have been demonstrated with a different architecture. ${ }^{45}$ NEP improvement, not just different operational wavelengths, is needed for GEP-S.

GEP KIDs are based on the MAKO design ${ }^{49}$ and adapted to work at shorter wavelengths. The MAKO design will work down to a wavelength of $\sim 100 \mu \mathrm{m}$, but below that modification will be required: the wire grid absorber linewidth used in the $350 \mu \mathrm{m}$ MAKO detectors is $0.4 \mu \mathrm{m}$. Scaling this design with wavelength would require prohibitively narrow lines for $\lambda<100 \mu \mathrm{m}$, even if using an ultraviolet stepper for film deposition. Our approach is to interrupt the absorber line with a meander that increases the resistance per unit length (and therefore the absorption efficiency), while simultaneously introducing a distributed capacitance to compensate for the accompanying inductance to tune the impedance. ${ }^{63}$ An impedance match to silicon may then be maintained with much wider and easier-to-fabricate lines.

This absorber design is shown in Fig. 8. For $10 \mu \mathrm{m}$ radiation, the linewidth is $200 \mathrm{~nm}$ and the unit cell is $2.4 \mu \mathrm{m}$ square, repeated continuously in the circular envelope of the absorber with electrical continuity meandered vertically. Simulations indicate that this geometry achieves a peak single-polarization absorptivity of just over $70 \%$ near $10 \mu \mathrm{m}$ for $R_{\square}=0.2$ to $0.8 \Omega$, bracketing the range expected for a $40-\mathrm{nm}$ Al film. Perido et al. ${ }^{63}$ presented dark measurements of 


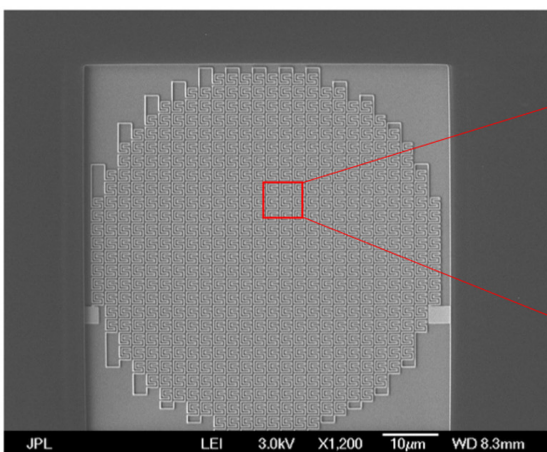

(a)

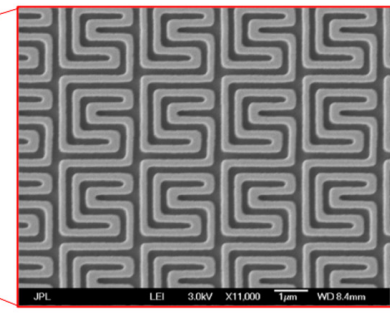

(b)

Fig. 8 A photograph the absorptive portion of a $\lambda=10 \mu \mathrm{m} \mathrm{KID,} \mathrm{courtesy} \mathrm{H.} \mathrm{G.} \mathrm{LeDuc,} \mathrm{reprinted} \mathrm{by}$ permission of the Creative Common license (creative commons.org/licenses/by/4.0/legalcode) from Fig. 1 of Ref. $64 .{ }^{63}$ (a) The inductor/absorber portion of the KID, which has a diameter of $60 \mu \mathrm{m}$. The unit cell size is $2.4 \mu \mathrm{m} \times 2.4 \mu \mathrm{m}$. The inductor is coupled to a larger interdigitated capacitor to form a microresonator. (b) Approximately 4 (horizontally) by 3 (vertically) unit cells. This absorber absorbs the vertical sense of polarization.

the KID array shown in Fig. 8. The frequency noise was dominated by two-level system noise, with typical values at $10 \mathrm{~Hz}$ of $S_{\mathrm{xx}}=(3-0.6) \times 10^{-17} \mathrm{~Hz}^{-1}$ at $T=100$ to $200 \mathrm{mK}$, consistent with the expected $T^{-1.7}$ scaling. Perido et al. ${ }^{63}$ also presented a resonant dual-polarization design for which simulations yield absorption of $70 \%$ in each polarization.

The pixel-to-pixel spacing is $300 \mu \mathrm{m}$, dominated in area by the resonators' interdigitated capacitors. Efficiently coupling the inductive absorbers to free-space radiation will be achieved with microlens arrays, and large feedhorn arrays are currently impractical for short mid-infrared wavelengths because manufacturing tolerances are too small. For minimal absorption by the substrates, the arrays should be fabricated with silicon for wavelengths $>20 \mu \mathrm{m}$ and germanium for 10 to $20 \mu \mathrm{m}$ because of the silicon absorption features in the 14 to $16 \mu \mathrm{m}$ range. Microlens arrays could either be fabricated separately and bonded to back-illuminated KID arrays ${ }^{64}$ or etched into the wafer frontside before or after KID fabrication. There are examples of silicon microlens fabrication in the literature that could achieve the micron-level tolerances required for operation down to $10 \mu \mathrm{m},{ }^{65-71}$ although those involving micromachining and laser etching would likely be too slow for arrays of $10^{4}$ detectors. A promising approach is the deposition of Fresnel "zone plate" microlens arrays on the wafer frontsides, which have already been demonstrated at $\lambda=10.6 \mu \mathrm{m}$ for antenna coupling. ${ }^{72}$ This approach has the virtue of fabrication simplicity but it rejects $50 \%$ of radiation. If noise-equivalent powers (NEPs) must be improved by a factor of $\sqrt{2}$, three-dimensional (3-D) hemispherical lenses or Fresnel lenses could be etched on the wafer frontsides instead of depositing Fresnel zones.

For all GEP focal planes, KIDs are organized into groups of $\sim 1500$ detectors that are spread across a 0.6 to $1.6 \mathrm{GHz}$ band and read out using electronics illustrated in Fig. 9. The choice of a 1.1-GHz center frequency resulted from a trade study in which smaller pixels were favored by the optical design but larger pixels reduced the readout frequency and bandwidth, and thus power dissipation. The readout electronics generate analog waveforms using RF-DACs that are transmitted to the cold focal plane, exciting all 1500 resonators. The 1-GHz bandwidth return signal from all $1500 \mathrm{KIDs}$ is digitized with RF-ADCs and digitally channelized with sufficient resolution to separate the individual KID frequencies. The KID readout scheme, initially demonstrated in $2006,{ }^{73}$ has now been implemented in various forms for ground-based and balloonborne instruments. $^{50,74-82}$

The GEP-I and GEP-S modules will share the same set of readout electronics (Fig. 9): they have separate, nonsimultaneous observing modes. Microwave switches on the readout lines enable selection of GEP-S or GEP-I for readout. There are 24 parallel readout channels available from three RF readout boards with eight channels each. The eight-channel RF readout cards share a payload electronics chassis with a chopping mirror driver card and two clock, processor, memory, and power cards for dual-string redundancy. The total estimated power consumption for the readout electronics is $484 \mathrm{~W}$. 


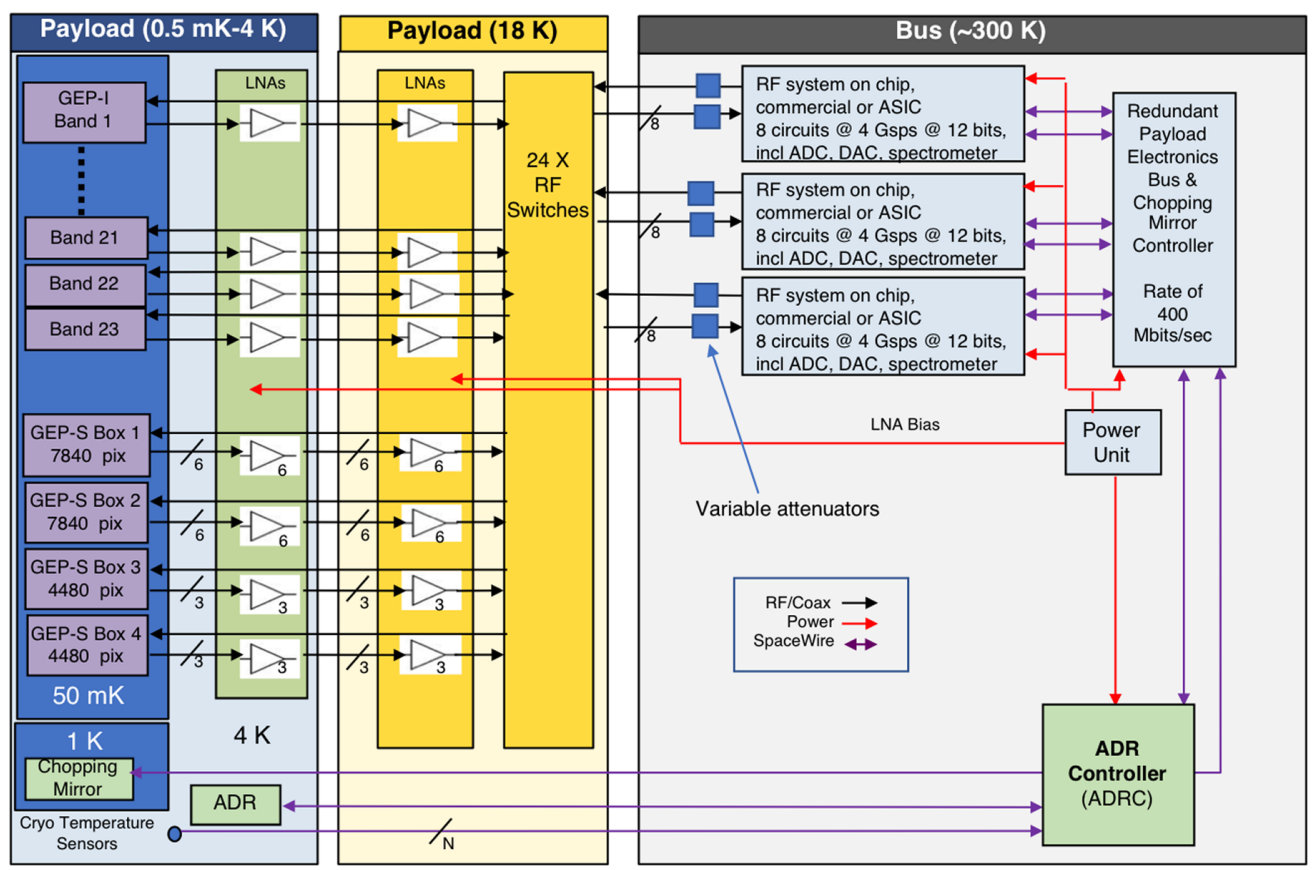

Fig. 9 Readout system for the GEP KID arrays. Electronics will be room temperature ( 300 K) except the amplifiers at 4 and $18 \mathrm{~K}$ and the KIDs themselves at $0.1 \mathrm{~K}$. The power consumption is estimated at $25 \mathrm{~W}$ per readout circuit. GEP-I uses 23 readout channels, GEP-S bands 1 and 2 use six readout channels each, and GEP-S Bands 3 and 4 use three readout channels each.

\subsection{KID Development and Readout Outlook}

For a launch date as early as January 1, 2029, technologies required for GEP must be at or above TRL 6 in 2025. Mid-infrared KIDs are currently at TRL 3, with the proof-of-concepts having been demonstrated. To reach TRL 6 , they must be validated at a component level in a relevant environment and in a subsystem operational environment. In this case, the latter indicates integration with optics and readout electronics. Here, we review the current state-of-the-art and itemize the technological improvements that must be made at a component level. This will have to followed by demonstrations of sensitivity, yield, and sufficiently low cosmic ray susceptibility for arrays while read out with flight-like electronics. Of the existing demonstrations shown in Fig. 7, the SPACEKIDs results ${ }^{45}$ are the closest to meeting the GEP requirements, which are listed in Table 2. By comparing the capability gap between GEP KID focal plane requirements and SPACEKIDs performance, specific advances required to achieve TRL 6 can be identified.

Table 2 Detector requirements for GEP versus achieved for SPACEKIDs. ${ }^{45}$ Notes: Minimum tile sizes for GEP-I/GEP-S shown; actual arrays could be multiples thereof. Tiles with $12 \times 120=$ 1440 pixel format are envisioned for GEP-I bands 1 to 18 . GEP-S bands 1 and 2 assume arrays with $112 \times 70$ format, which could consist of tiles with $28 \times 35=980$ pixels. The dynamic range is specified for a 1-Jy calibration source, e.g., an asteroid. ${ }^{45,83}$ Techniques to mitigate electrical and optical crosstalk, and cosmic ray susceptibility, have been demonstrated. ${ }^{45,84,85}$

\begin{tabular}{|c|c|c|c|c|c|c|c|c|c|c|c|}
\hline & $\begin{array}{c}\text { Tile } \\
\text { size } \\
\text { (pixels) }\end{array}$ & $\begin{array}{c}\lambda \\
(\mu \mathrm{m})\end{array}$ & $\begin{array}{c}\text { NEP } \\
\left(\mathrm{WHz}^{-1 / 2}\right)\end{array}$ & $\begin{array}{c}\text { MUX } \\
\text { (pix/ } \\
\mathrm{GHz})\end{array}$ & $\begin{array}{l}\text { Pitch } \\
(\mu \mathrm{m})\end{array}$ & $\begin{array}{l}\tau_{\mathrm{det}} \\
(\mathrm{ms})\end{array}$ & $\begin{array}{l}\text { Min. } \\
\text { yield }\end{array}$ & $\begin{array}{c}\text { Dynamic } \\
\text { range }\end{array}$ & $\begin{array}{l}\text { Cross- } \\
\text { talk } \\
\text { (dB) }\end{array}$ & $\begin{array}{c}1 / f \\
\text { knee } \\
(\mathrm{Hz})\end{array}$ & $\begin{array}{c}\text { Cosmic } \\
\text { ray } \\
\text { deadtime }\end{array}$ \\
\hline SPACEKIDs & 961 & 350 & $3 \times 10^{-19}$ & 240 & 1600 & 1.5 & $83 \%$ & $10^{5}$ & -30 & 0.2 & $5 \%$ \\
\hline GEP-I & 1440 & $\begin{array}{c}10 \text { to } \\
400\end{array}$ & $7 \times 10^{-19}$ & 1500 & 300 & $<4$ & $80 \%$ & 5,000 & -17 & $<1$ & $<2 \%$ \\
\hline GEP-S & 980 & $\begin{array}{c}24 \text { to } \\
193\end{array}$ & $1 \times 10^{-19}$ & 1500 & $300 \times 600$ & $<4$ & $80 \%$ & 1,000 & -17 & $<1$ & $<2 \%$ \\
\hline
\end{tabular}




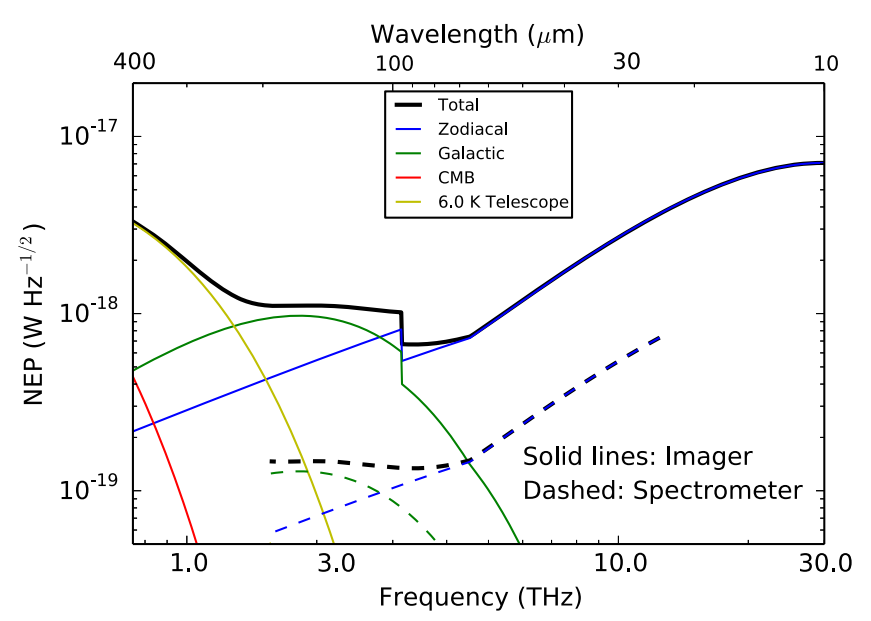

Fig. 10 Detector NEPs for various photon backgrounds for the zodiacal light, the Galactic thermal dust emission, and GEP telescope, and instrument parameters. This shows why a $T_{\text {tele }} \leq 6 \mathrm{~K}$ telescope is necessary: it is not possible to achieve astrophysical photon background-limited performance for wavelengths longer than $200 \mu \mathrm{m}$ with $T_{\text {tele }}>6 \mathrm{~K}$. A cooler telescope would have better GEP-I sensitivity, but it would be of limited value because the astrophysical source confusion is expected to be severe for $\lambda \geq 200 \mu \mathrm{m}$. GEP-S would benefit from cooling the telescope to $4 \mathrm{~K}$ even at the longest wavelengths, so this possibility may be adopted later.

1. Sensitivity: To be astrophysically photon background limited, which results in the best possible sensitivity, the telescope and optics must be below $6 \mathrm{~K}$ and the detector NEPs must be below the quadrature sum of all the other NEP terms (Fig. 10): $7 \times 10^{-19} \mathrm{~W} \mathrm{~Hz}^{-1 / 2}$ for GEP-I and $1 \times 10^{-19} \mathrm{~W} \mathrm{~Hz}^{-1 / 2}$ for GEP-S. GEP-S requires at least a factor of three improvement in NEP over SPACEKIDs. This can be achieved through reducing the detector active volume (thereby increasing the quasiparticle density and responsivity) below the $\sim 100 \mu \mathrm{m}^{3}$ used by SPACEKIDs.

2. Multiplexing: The minimum detector-multiplexing factor for GEP is 1500 pixels per GHz of readout bandwidth. This $6 \times$ increase in multiplex factor relative to SPACEKIDs is based on a $6 \times$ reduction in readout frequency, from 6 to $1 \mathrm{GHz}$ (GEP's microwave readout band center). Multiplex factors of 6500 per $\mathrm{GHz}$ have been demonstrated at $200 \mathrm{MHz}$ readout frequency.$^{50}$ Further improvements have been demonstrated using postfabrication resonator trimming methods. ${ }^{86,87}$

3. Pitch: The baseline GEP-I pixel pitch is $300 \mu \mathrm{m}$ from wavelengths of 10 to $60 \mu \mathrm{m}$ to provide $3.43^{\prime \prime}$ pixel sizes (the pitch is larger for $\lambda>60 \mu \mathrm{m}$ ). For GEP-S, the pixel pitch is $300 \times 600 \mu \mathrm{m}$ to meet the spectral resolution requirement. The GEP requirements can be met with the design in Fig. 8 and microlens arrays. Smaller pixels have smaller inductors and area-dominating interdigitated capacitors, and therefore higher resonance frequencies, which presents an engineering design trade space of pitch versus readout frequency.

To date, all KID instrument readout systems use field-programmable gate arrays (FPGAs) ${ }^{88}$ for the digital channelization. This channelization is conceptually equivalent to an FFT and sometimes is implemented as such. ${ }^{74,80}$ First-generation KID readouts ${ }^{50,75-77}$ typically used Virtex $-5^{89}$ hardware to process a $500-\mathrm{MHz}$ bandwidth using about $50 \mathrm{~W}$, across which up to 4000 KIDs could be multiplexed. ${ }^{50}$ Similarly, the CORE mission study ${ }^{90}$ concluded that a 1-GHz bandwidth could be read with $50 \mathrm{~W}$ using existing space-grade, TRL-9 Virtex-5 FPGAs. ${ }^{91}$ Newer-generation FPGAs could have $10 \times$ lower power consumption, ${ }^{92}$ (Fig. 11), thus the laboratory figure of $50 \mathrm{~W}$ per $1 \mathrm{GHz}$ channel is conservative, and there is strong interest in the use of advanced FPGAs in space. ${ }^{98-103}$ Radiation test results on late-generation FPGAs have been positive. ${ }^{91,104-106}$ As a result, Xilinx's 20-nm KU060 FPGA (used in the SMURF readout electronics developed at SLAC) $)^{82}$ will be available as a space-rated product by late $2020 .{ }^{91}$ This bodes well for even more advanced options, such as the new 16-nm FinFET Xilinx RF-system 


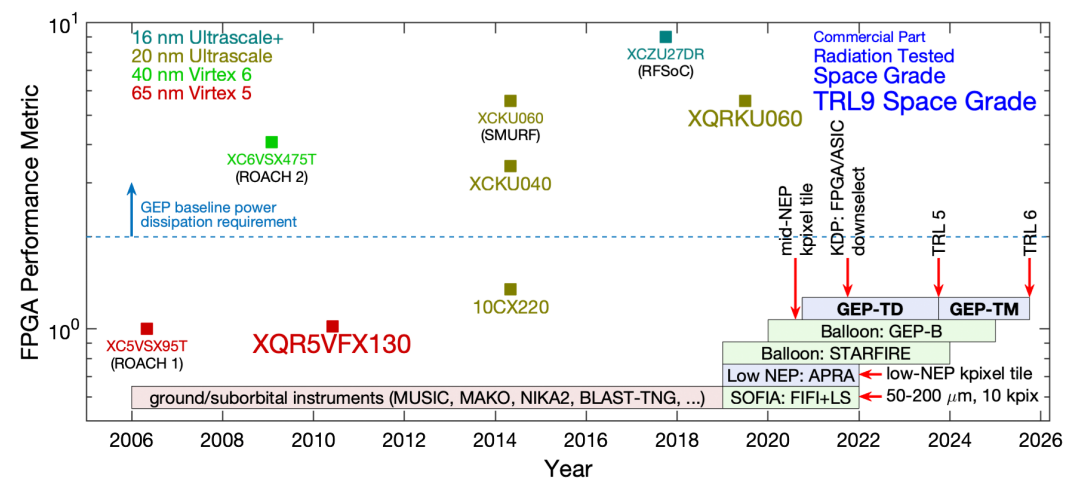

Fig. 11 As part of the GEP concept design study, a plan was developed to mature KIDs and payload electronics technologies in advance of an anticipated Probe-class proposal through laboratory (shaded in red), SOFIA and balloon-borne instruments (green), and system-level demonstrations (blue) that started in 2019. In the top of the figure, feasibility of GEP readout electronics is illustrated using the evolution of Xilinx FPGA capability over time. Specific devices are plotted as points labeled by the part number, with data sheets listed in the references. The horizontal axis gives the introduction date, and the vertical axis is a composite performance metric that combines the number of logic gates and DSP cells, memory, and IO capability. The dashed blue line indicates the approximate minimum capability required for meeting the GEP baseline power dissipation of $25 \mathrm{~W}$ per $1 \mathrm{GHz}$ readout channel. Color is used to indicate the CMOS technology node, while font size indicates suitability for use in the space radiation environment. The XQRKU060, which Xilinx plans to release as a space-grade part in 2020, is a strong candidate. GEP's technology development plan calls for a TRL 6 validation of all hardware required for GEP's payload by 2025. FPGA data sheets are listed in Refs. 92-97.

on chip (RFSoC), which integrates eight ADCs, eight DACs, and considerable FPGA logic into a single chip; the entire GEP readout could potentially be reduced to one or two such chips. A board with this chip has been released; ${ }^{107}$ initial power dissipation estimates are well below the GEP conservative assumption of $25 \mathrm{~W}$ per $1 \mathrm{GHz}$ channel. Alternatively, mixed-signal application-specific integrated circuits that integrate ADCs and signal processing have been developed for similar applications. ${ }^{108}$

\section{GEP Surveys, Simulations, and Science}

\subsection{Surveys}

GEP is designed to do large, unbiased surveys. Its overarching goal is to provide a legacy dataset with broad utility for studying the evolution of star-formation, interstellar medium, and black hole growth in galaxies. Parameters of GEP's surveys in the context of other surveys are shown in Figs. 12 and 13 with complementary surveys and the astrophysics that they probe.

The GEP-I survey program is optimized to sample a comprehensive range of redshifts and galaxy luminosities (Table 3). A combination of four depths and areas will sample low redshift and rare, luminous galaxies, faint, high-redshift galaxies, and intermediate redshift and luminosity galaxies. Large numbers of galaxies will be detected in each tier, preventing sample variance and small number statistics from hampering conclusions on galaxy evolution with redshift (Fig. 14). It is anticipated that the GEP-I surveys (except the all-sky survey) will be centered on and divided between the north and south ecliptic poles to minimize the photon backgrounds from primarily zodiacal dust and secondarily Galactic dust. This will provide sky coverage overlap with Euclid and Nancy Grace Roman Space Telescope (NGRST) surveys, which will provide near-infrared photometry, morphologies, and stellar masses for galaxies detected by GEP.

Three types of spectroscopic measurements with GEP-S will complement GEP-I photometric surveys: (1) individual observations of specific galaxies identified in the GEP-I surveys to provide precise redshifts and to validate PAH redshifts and to obtain measurements of the farinfrared emission lines. (2) Unbiased spectroscopic surveys obtained by rastering GEP-S on the 


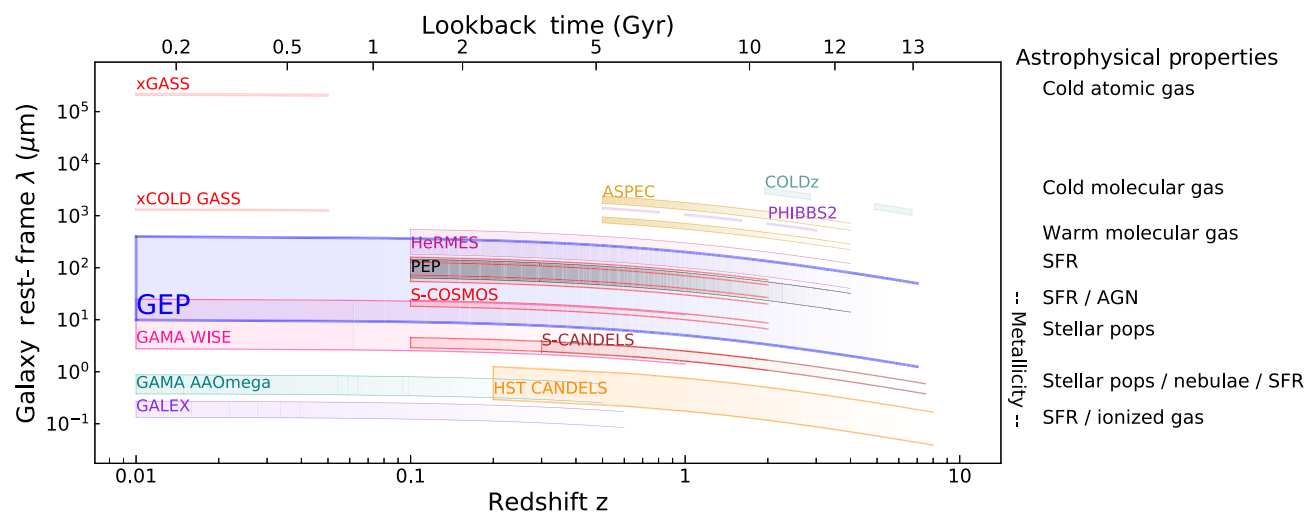

Fig. 12 Context for galaxy evolution surveys: a representative sample of galaxy surveys as a function of wavelength and redshift. ${ }^{109-120}$ The emphasis is on space-based surveys and not all surveys are shown to minimize confusion in the figure. Some ground-based surveys are included that provide critical, unique information. Completed or nearly completed surveys have solid borders, while planned surveys have dashed borders. The left terminations indicate either lower redshift limits arising from observational spectral bands or are indicative of the areas surveyed. For example, WISE covered the entire sky and therefore probed very low redshifts comprehensively, whereas deep, narrow surveys such as HST CANDELS and Spitzer CANDELS probed fewer galaxies at the lowest redshifts. Terminations that fade to the right indicate reduced sensitivity to detecting galaxies at high redshifts because of flux limits.

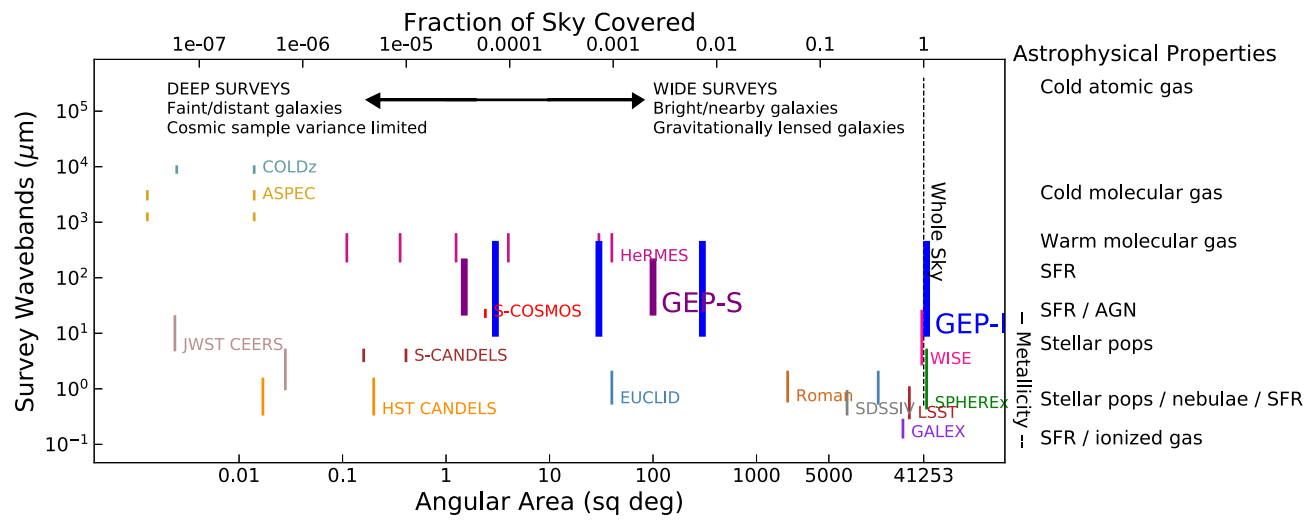

Fig. 13 Context for galaxy evolution surveys: a representative sample of galaxy surveys as function of wavelength and sky area. ${ }^{109-112,114,115,119-126}$ Surveys on the left side of the panel are generally deep, optimized for detecting faint and high-redshift galaxies but are cosmic sample variance limited, whereas wide surveys toward the right generally detect brighter and lowerredshift galaxies (the exception is lensed galaxies, which are rare). GEP-I and GEP-S surveys indicated with the bold purple and blue lines occupy a broad range of wavelength and sky area space. IRAS covered the entire sky, but its observations were shallow (by comparison to GEP) and did not include spectra. GEP surveys extend sensitive mid- to far-infrared imaging and spectroscopy more than two orders of magnitude from previous surveys, finally exploring the dusty side of galaxy evolution across the whole sky and over two-third the age of the Universe.

sky. (3) Spectral maps of nearby galaxies. GEP-S will perform a deep spectroscopic survey over 1.5 square degrees and a wide spectroscopic survey over 100 square degrees (Table 3 ). The spectral survey datasets will detect galaxies the far-infrared fine-structure transitions (and the continuum, when binned). The wide survey will be used to stack GEP spectra on the NGRST and/or Euclid grism sources to provide high signal-to-noise ratio average galaxy spectra in bins (Table 4). The GEP-S spectral mapping speed is shown in Fig. 15, where it is seen that GEP would be a substantial improvement of the state of the art with times to survey sky regions in the far-infrared six orders of magnitude faster than previous observatories. 
Table 3 GEP galaxy surveys and yields obtainable with background-limited sensitivities.

\begin{tabular}{|c|c|c|c|c|c|}
\hline Module & Area & Depth & Region & No. galaxies & $\begin{array}{l}\text { No. } \\
\text { redshifts }\end{array}$ \\
\hline GEP-I & $3 \mathrm{sq}$ deg & $10 \mu \mathrm{Jy}$ & Ecliptic poles & $10^{6}$ above $\sigma_{\text {conf }}$ & $10^{5}$ \\
\hline GEP-I & $30 \mathrm{sq}$ deg & $30 \mu \mathrm{Jy}$ & Ecliptic poles & $10^{6}$ above $\sigma_{\text {conf }}$ & $2 \times 10^{5}$ \\
\hline GEP-I & $300 \mathrm{sq}$ deg & $100 \mu \mathrm{Jy}$ & Ecliptic poles & $10^{7}$ above $\sigma_{\text {conf }}$ & $5 \times 10^{5}$ \\
\hline GEP-I & 300 All sky & $1 \mathrm{mJy}$ & - & $10^{8}$ above $\sigma_{\text {conf }}$ & $10^{6}$ \\
\hline GEP-S & $1.5 \mathrm{sq}$ deg & $\begin{array}{c}7 \times 10^{-20} \mathrm{~W} \mathrm{~m}^{-2} \\
@ 100 \mu \mathrm{m}\end{array}$ & Ecliptic poles & $\begin{array}{c}2 \times 10^{4} \text { for } \\
1<z<2\end{array}$ & $2 \times 10^{4}$ \\
\hline GEP-S & $\begin{array}{l}\text { Local galaxy } \\
\text { mapping }\end{array}$ & $\begin{array}{c}1 \times 10^{-10} \mathrm{~W} \mathrm{~m}^{-2} \mathrm{ster}^{-1} \\
@ 122 \mu \mathrm{m}\end{array}$ & Distributed & 400 & $\mathrm{~N} / \mathrm{A}$ \\
\hline \multirow[t]{2}{*}{ GEP-S } & $100 \mathrm{sq}$ deg & $\begin{array}{c}3.5 \times 10^{-19} \mathrm{~W} \mathrm{~m}^{-2} \\
@ 100 \mu \mathrm{m}\end{array}$ & $\begin{array}{l}\text { Overlap NGRST/ } \\
\text { Euclid grism fields }\end{array}$ & $4 \times 10^{5}$ & $5 \times 10^{4}$ \\
\hline & & & Intensity mapping & - & - \\
\hline GEP-S & $\begin{array}{l}\text { GEP-I galaxies } \\
1.2<z<3\end{array}$ & $3 \times 10^{-20} \mathrm{~W} \mathrm{~m}^{-2}$ & Distributed & 300 & 300 \\
\hline
\end{tabular}

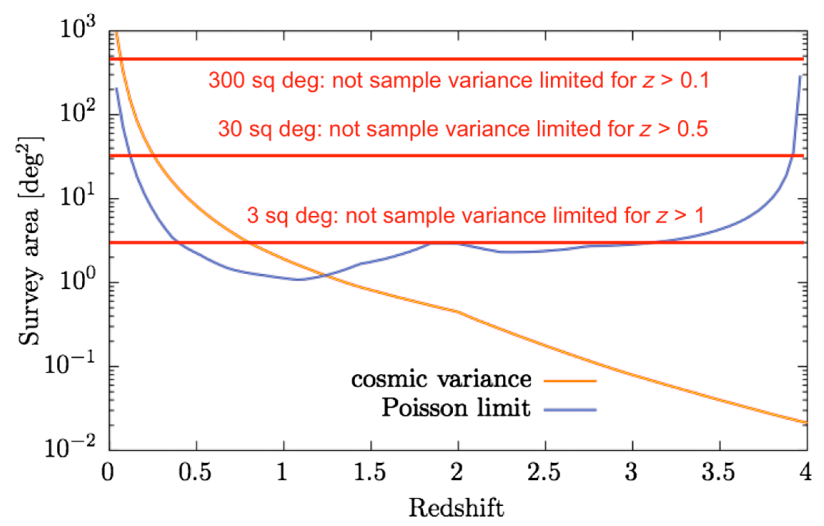

Fig. 14 Cosmic sample variance and the Poisson limit for GEP surveys. The red horizontal lines denote the depths of three of the four survey layers (excluding the all-sky survey). GEP's "wedding-cake" survey layers observe sufficiently large angular areas that they will not be limited by cosmic sample variance in the target redshift ranges of each tier. The 3- and 30-square degree surveys will also not be limited by shot noise for $0.5 \leq z \leq 3.5$. The 300 square degree has a lower shot noise limit $(z \sim 0.1)$ and the all-sky survey will extend to even lower redshifts. The blue line shows the minimum areas needed to detect enough galaxies to enable luminosity function slope measurements to a precision of $10 \%$ at the faint end.

Table 4 GEP stacking of IR galaxy datasets obtainable with background-limited sensitivities.

\begin{tabular}{lcccccccc}
\hline \hline Survey & $\begin{array}{c}\text { Redshift } \\
\text { of } \mathrm{H} \alpha\end{array}$ & $\begin{array}{c}\text { Area } \\
\text { (sq. } \\
\text { deg) }\end{array}$ & $\begin{array}{c}\text { Flux depth } \\
\left(\mathrm{erg} / \mathrm{s} / \mathrm{cm}^{2}\right)\end{array}$ & $\begin{array}{c}\text { Density } \\
\left(\mathrm{deg}^{-2}\right)\end{array}$ & $\begin{array}{c}\text { Median } \\
\text { SFR } \\
\left(\mathrm{M}_{\odot} / \mathrm{yr}\right)\end{array}$ & $L_{\mathrm{IR}}\left(L_{\odot}\right)$ & $N_{\text {Total }}$ & Stacks \\
\hline Euclid & 0.9 to 1.8 & 15,000 & $2.4 \times 10^{-16}$ & 4000 & 33 & $1.9 \times 10^{11}$ & 60 million & 260 \\
NGRST & 1 to 2 & 2200 & $1.0 \times 10^{-16}$ & 10,000 & 15 & $6.8 \times 10^{10}$ & 22 million & 950 \\
\hline \hline
\end{tabular}




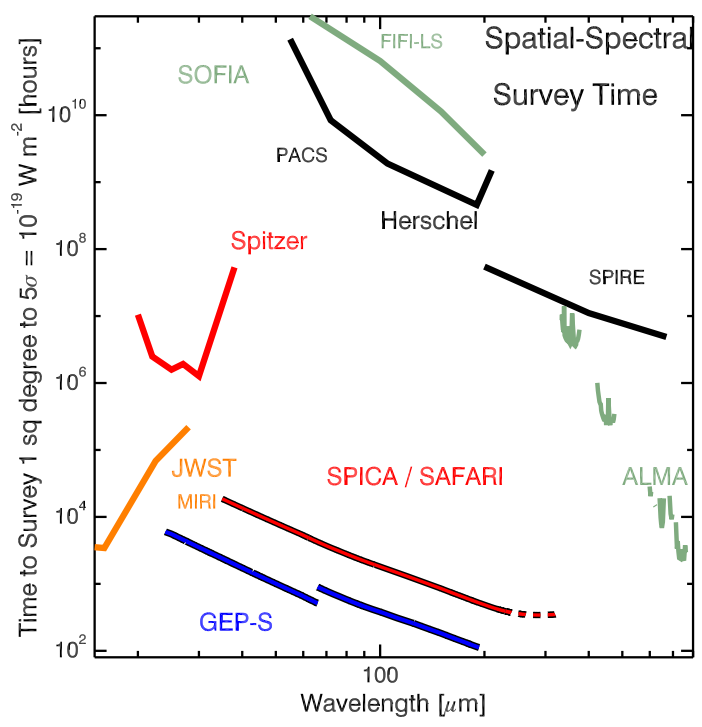

Fig. 15 Spectral survey time to a given depth in the mid- and far-IR (lower is faster). The GEP-S spectrometer modules offer gains of six orders of magnitude in observing speed relative to stateof-the-art-Herschel and SOFIA-because of their warm apertures. The GEP-S speed exceeds that of SPICA because of the larger focal plane array format that enables long slits.

\subsection{Theoretical Framework and Simulations}

To quantify the science yield of the GEP-I survey program, a mock survey was constructed using a combination of the Millennium $\mathrm{N}$-body simulation ${ }^{127}$ to provide the distribution of large-scale structure and the Galacticus semianalytic model ${ }^{128}$ to populate that simulation with galaxies based on physical models. For each dark matter halo in the simulation volume, the star-formation rate and black hole accretion rate of the galaxy were computed. Bolometric infrared luminosities from star-formation were estimated as $L_{\mathrm{IR}}=2.6 \times 10^{45}\left(\mathrm{SFR} / M_{\odot} \mathrm{yr}^{-1}\right)$ ergs s${ }^{-1}{ }^{129}$ and those due to AGN activity as $\epsilon \dot{M}_{\bullet} c^{2}$ (where $\epsilon$ is the radiative efficiency computed by Galacticus from the black hole spin and accretion rate, and $\dot{M}_{\bullet}$ is the black hole accretion rate). Infrared spectra with the corresponding AGN fraction were then assigned to galaxies using the models of Dale et al. $^{28}$ and normalized to give the computed total infrared bolometric luminosity. In the Dale et al. ${ }^{28}$ models, the distribution of dust heating intensities (and, therefore, temperatures) is parameterized in terms of $\alpha_{\mathrm{SF}}$, the exponent of the power-law distribution of heating intensities. Values of $\alpha_{\mathrm{SF}}$ for model galaxies were drawn from a Gaussian distribution with mean, $\bar{\alpha}_{\mathrm{SF}}=1.75$, and dispersion, $\sigma_{\alpha_{\mathrm{SF}}}=0.25$, with no redshift dependence. A range of different possible values of $\bar{\alpha}_{\mathrm{SF}}$ and $\sigma_{\alpha_{\mathrm{SF}}}$ was explored, with the above values selected as those which result in the closest match between our simulated and observed numbers counts of galaxies over the 24- and 350- $\mu \mathrm{m}$ wavelength range. ${ }^{130,131}$ These spectra were then used to compute broadband luminosities for model galaxies in each GEP-I band. Finally, a light cone from this synthetic catalog was extracted, corresponding to a 4-square degree area, from $z=0$ to 3 , and observed fluxes were determined in all GEP-I bands for each galaxy.

The galaxy number counts estimated in this way are lower limits because these (and other) galaxy evolution models generically underpredict the number of very high luminosity galaxies ${ }^{132}$ $\left(\sim 10^{13} L_{\odot}\right)$. Although we attempted to tune the Galacticus model number counts to match observations from Spitzer and Herschel by judiciously choosing from the Dale et al. ${ }^{28}$ spectral library, at high flux densities (i.e., $>1 \mathrm{mJy}$ at $160 \mu \mathrm{m}$ ) the model counts are almost an order of magnitude too low compared with observations. ${ }^{131}$ The agreement is much better at 24 and $70 \mu \mathrm{m}$, coming close to matching observations. ${ }^{130}$ Some of the bright observed galaxies that are not accounted for in the models likely result from gravitational lensing, although lensing is unlikely to account for the majority of the discrepancy. We adopt our model predictions for the detection rates with the understanding that they are likely conservative. 


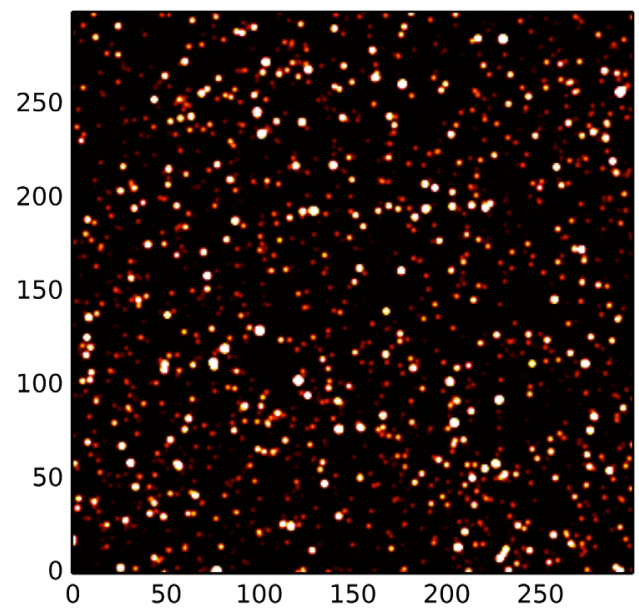

Fig. 16 A $10-\mu \mathrm{m}$ still image from Video 1, a rendering of the Galacticus simulations. The axis units are pixel number, with $3.43^{\prime \prime} \times 3.43^{\prime \prime}$ pixels-the image is $17.2^{\prime}$ on a side. The animation runs continuously through the bands from 10 to $80 \mu \mathrm{m}$ showing that galaxies alternately brighten and dim as PAH emission lines move through the bands and can thus be used to measure redshifts (Video 1, MP4, 3.5 MB [URL: https://doi.org/10.1117/1.JATIS.7.3.034004.1]).

Noise was added to the simulated maps corresponding to the expected map depths after they were convolved with GEP beam sizes. A small region of the maps stepping through the bands is animated in Fig. 16.

\subsection{Extragalactic Source Confusion}

Extragalactic source confusion arises when images of galaxies-the point spread function for unresolved galaxies-overlap. It happens at far-infrared wavelengths where diffraction-limited beams can be several arcseconds or larger. Confusion "noise" is the uncertainty in the extraction of a given source's flux due to the presence of a background of other sources which cannot accurately be subtracted from the signal. Analytically, the confusion noise $(1 \sigma)$ is just the standard deviation of the flux from beam to beam, and for extraction of sources from a map, a "confusion limit" arises at $3-5 \times$ this $1 \sigma$ value. This limiting flux below which extractions are unreliable typically corresponds to that for which density of sources is 1 per several $(\sim 10)$ beams, depending on the source count relation. This basic paradigm holds true when referring to extracting sources from a map without prior information, but there are well-developed methods to employ prior higher-resolution datasets to extract source parameters in a larger far-IR beam. An excellent example is use of Spitzer IRAC and MIPS $24 \mu \mathrm{m}$ positions to extract (statistically) fluxes in the 70 and $160 \mu \mathrm{m}$ maps. ${ }^{133}$ In this section, we assess the important issue of source confusion with the GEP-I datasets, but first note that spectral surveys with GEP-S should not suffer appreciably from source confusion.

For spectral mapping with GEP-S, source confusion should not limit the extraction of line intensities. The key point is that the bright mid- and far-IR spectral lines are sparse and well-separated spectrally, and they form an unambiguous template for redshift identification. Thus, each line can be conclusively identified and measured, even with multiple line emitting sources in the beam. For a 3-D spatial-spectral survey, the analog of source counts for twodimensional is line counts, the number of line emitters above a given flux level in a typical spatial-spectral bin of the survey. This issue was studied for the OST, which also features a wideband, moderate-resolving-power spectrograph; Ref. 134 is a good reference. As an example they predict that at $190 \mu \mathrm{m}$ (which is approximately GEP-S long-wavelength range limit), the density of lines with fluxes above $10^{-20} \mathrm{~W} \mathrm{~m}^{-2}$ (corresponding to a 25-h integration with GEP-S) per beam-bin is $1 / 150$ for Origins. Correcting this for the larger GEP beam $(2 / 5.9)^{-2}$ and slightly smaller resolving power $(200 / 300)^{-1}$ results in an estimate of one source per 12 beam-bins for GEP-S at this wavelength. This is approaching but not exceeding 


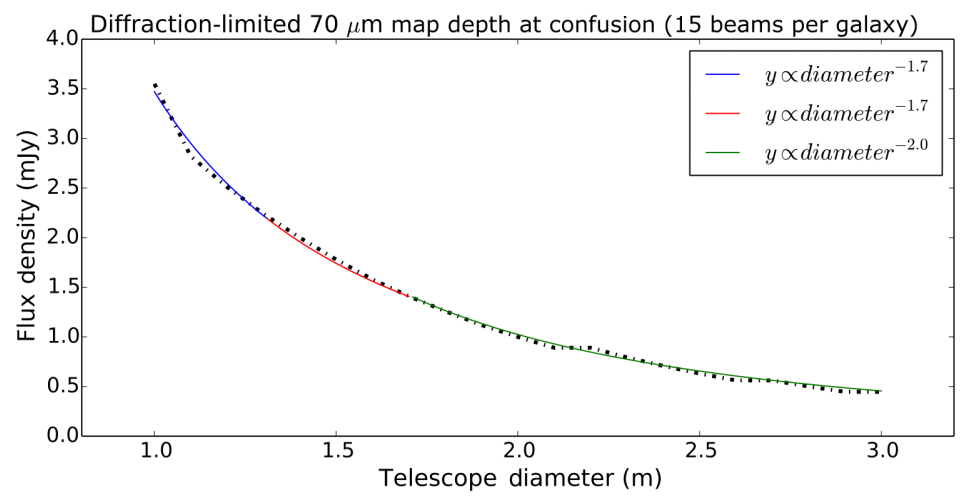

Fig. 17 The flux density for 15 beams per galaxy as a function of telescope aperture diameter at $\lambda=70 \mu \mathrm{m}$. The number counts are from Ref. 139 and the curves are piecewise fits. GEP's $2.0 \mathrm{~m}$ aperture is a "sweet spot" between rapidly worsening confusion for smaller apertures and increasing cost for larger apertures.

a practical confusion limit. Shorter wavelengths of course are more forgiving with the smaller beam.

We estimate the extragalactic confusion noise to be expected for GEP-I observations by considering confusion noise measurements from previous observations. The confusion noise flux density was measured—or upper limits were placed—with Spitzer 70 and $160 \mu \mathrm{m}^{135,136}$ and Herschel 70, 100, and $250 \mu \mathrm{m} .{ }^{131,137,138}$ We start by deriving an empirical relationship for confusion noise as a function of telescope aperture diameter to assess the dependence of confusion on telescope size and to check for consistency between Spitzer and Herschel observations, which had different wavebands and aperture sizes ( 0.85 and $3.5 \mathrm{~m}$, respectively). Using the number counts models of Béthermin et al., ${ }^{139}$ the $\lambda=70 \mu \mathrm{m}$ flux density corresponding to 15 beams per source (where galaxies are not be substantially blended) scales approximately as the inverse of aperture diameter squared ( $\propto D^{-2}$, slightly steeper for apertures smaller than $1.7 \mathrm{~m}$, Fig. 17). Confusion noise measurements in the literature cited above scaled by this relationship are consistent, which validates the scaling relation for interpolation to the GEP $2.0 \mathrm{~m}$ aperture for estimation of confusion noise. Critically, the scaling also shows that aperture diameters below $2.0 \mathrm{~m}$ will be increasingly susceptible to confusion. Since the confusion flux density drops slowly with telescope diameter beyond $2.0 \mathrm{~m}, 2.0 \mathrm{~m}$ represents something of a "sweet spot" in the trade between confusion and cost.

A comparison of the expected GEP-I survey map depths for a 2.0-m aperture with the confusion measurements cited in the literature already mentioned yields the following:

1. At $\lambda=24 \mu \mathrm{m}$, GEP-I will not be confusion limited.

2. Astrophysically background-limited $\lambda=70 \mu \mathrm{m}, 1 \sigma$ RMS GEP-I map depths are 6, 20, 60, and $6 \mathrm{mJy}$ for the 3, 30, 300 square degree, and all-sky surveys, respectively. Scaling the Spitzer $300 \mu \mathrm{Jy}, \lambda=70 \mu \mathrm{m}$ confusion noise by the empirical $D^{-2}$ relation yields $50 \mu \mathrm{Jy}$ RMS. Thus, the noise in the deepest two surveys will be dominated by confusion noise at $\lambda=70 \mu \mathrm{m}$, the observational noise will just reach the confusion noise in the 300 square degree survey, and the all-sky survey will not be strongly affected by confusion noise.

3. At $100 \mu \mathrm{m}$, scaling by the $D^{-2}$ relation, Herschel's observed confusion noise of $200 \mu \mathrm{Jy}$ RMS would be $600 \mu \mathrm{Jy}$ for GEP-I's $2.0 \mathrm{~m}$ aperture. Thus, the all-sky survey, with a $1 \sigma$ map depth of $600 \mu \mathrm{Jy}$, would just reach the confusion noise level.

4. All four surveys will likely reach the confusion noise level at wavelengths longer than $\lambda=100 \mu \mathrm{m}$. However, the observations should be made at these wavelengths to measure total luminosities of bright, low- $z$ galaxies, and lensed high- $z$ galaxies.

In short, GEP-I will likely have significant confusion noise at $\lambda=70 \mu \mathrm{m}$ and longer, but not at shorter wavelengths. GEP-I will have to integrate deeper than the $70 \mu \mathrm{m}$ confusion noise in the two deepest surveys for PAH redshifts of $z \leq 4$ galaxies with the wavebands at $50 \mu \mathrm{m}$ and below, which will not be limited by confusion noise. In addition, monochromatic fluctuation "probability 


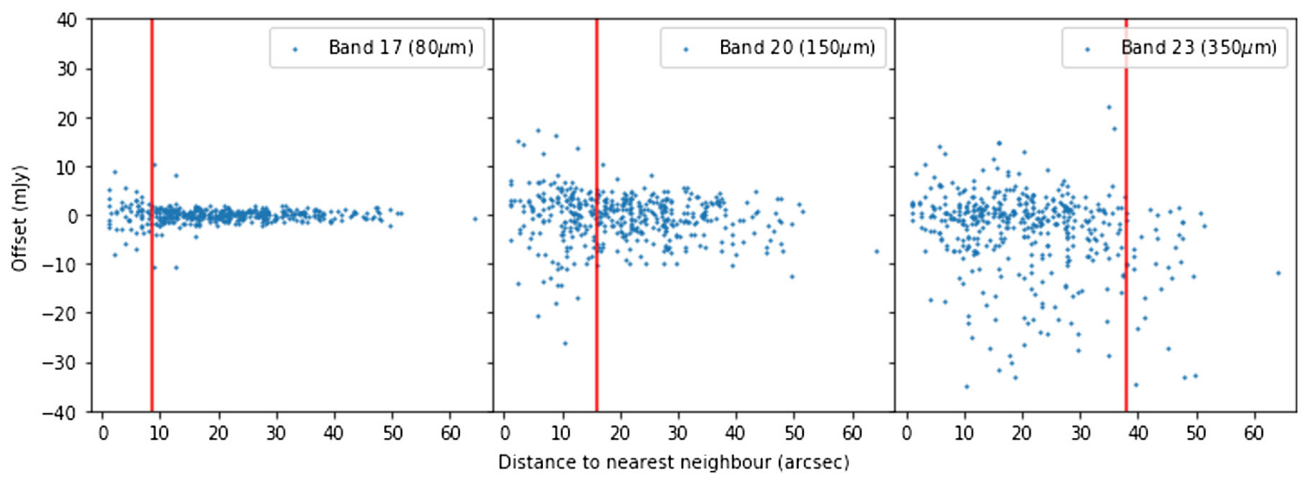

Fig. 18 Differences between XID+ fitted and true galaxy flux densities as a function of distance to the nearest galaxy neighbor for three GEP-I long-wavelength bands: 17, 20, and 23 (with FWHM beam sizes of $8.6^{\prime \prime}, 16^{\prime \prime}$, and 38", respectively). Red vertical lines show the beam width as measured by the full width at half maximum. For bands 17 and 20, the fractional error in the flux density is small down to the beam size and clustered around zero. At the beam size and below the dispersion increases, although there is no bias. For band 23, GEP-l's longest-wavelength band, the simulation data do not extend to great enough separations to see that the offset decreases toward zero for separations above the beam size. In this band, there is a negative bias for some galaxies that will have to be corrected.

of deflection" $\mathrm{P}(\mathrm{D})$ analyses show that it is possible to constrain galaxy populations meaningfully with observations deeper than the confusion noise level. ${ }^{140,141}$ A polychromatic $\mathrm{P}(\mathrm{D})$ analysis with GEP-I observations covering the redshifted PAH features would be extremely powerful; it would yield precise galaxy number counts and redshifts statistically for the ensemble, and therefore luminosity functions, and tightly constrain galaxy evolution models. Furthermore, using cross-identification with counterparts at shorter wavelengths, galaxy properties can be measured even when there is source confusion. For example, Labbe et al. ${ }^{142}$ showed that contamination by confusion can be reduced a factor of six with short-wavelength prior-based photometry, and positional and flux priors from ancillary data could enable improved spectral energy distribution extraction. In addition, because the detector count in wavebands longer than $100 \mu \mathrm{m}$ (GEP-I band 19) is small (4.2) compared with shorter wavelengths, the cost of retaining them is merited for measurements of far-infrared luminosities, for nearby galaxy science that do not require observations as deep, and for $\mathrm{P}(\mathrm{D})$-type fluctuation analyses.

For wavelengths $\lambda \geq 70 \mu \mathrm{m}$, corresponding to GEP-I bands 17 to 23 , photometry of partly blended sources can still be extracted reliably. The Next Generation (X)Cross Identification (XID+) code was developed to estimate flux densities accurately from confusion-limited Herschel imaging, ${ }^{143}$ we apply it here to GEP-I. The performance of XID+ was quantified by measuring the differences between fitted and true galaxy flux densities as a function of the distance to the nearest galaxy neighbor using the simulations described in Sec. 5.2. Positional priors were assumed from the mid-infrared wavebands and the fitted galaxy far-infrared flux densities were compared to their true values. Figure 18 shows that down to galaxy separations of the beam size-below the classical confusion "limit"—galaxy flux densities can be deblended with small fractional errors and little or no bias in most cases. Using the deblended sources, the map flux densities were reproduced with small residuals, although with poorer performance at the map edges because of missing galaxies outside the field of view. This will not be a concern for the majority of galaxies detected in GEP-I surveys, for which the minimum size is 3 square degrees.

\subsection{Anticipated Science}

\subsubsection{GEP-I Redshift Measurements}

To measure the cosmic star formation history of the Universe, GEP requires redshifts with precision $\sigma_{z} /(1+z) \leq 10 \%$ to $z=2$, a requirement that our simulations show is achieved with 
margin. In general, the redshift uncertainty is set by the width of the GEP-I bands relative to the widths of the PAH features.

Expected GEP-I redshift precision was estimated by adding noise to the model spectra according to each of the map depths given by the photon backgrounds. The mock spectra were binned into the GEP-I bands and $\chi_{\nu}^{2}$ were calculated by comparison to the spectral model and two other different spectral models: since the spectra of galaxies will not be known a priori, the comparison models were used to ascertain the uncertainties incurred by having a spectrum different from the model. Only the first 11 GEP-I bands (10 to $37 \mu \mathrm{m}$ ) were used for the redshift measurements because the steeply rising mid-infrared dust spectra influence the redshifts and the dust temperatures will not be known a priori. A sample GEP-I redshift chi-squared distribution is shown in Fig. 19. The nominal model had strong PAH emission features and the alternate comparison models had: (1) strong PAH emission features but cooler dust (hence a more slowly rising spectrum with wavelength) and (2) hot dust that substantially overwhelmed the PAH features above $10 \mu \mathrm{m}$. The results were as follows:

1. Redshifts are obtainable for $10^{11} L_{\odot}$ galaxies (corresponding to a median stellar mass of $\left.4 \times 10^{9} \mathrm{M}_{\odot}\right)$ out to $z=2$ in the 3 square degree survey with $\sigma_{z} /(1+z) \leq 0.1$.

2. Redshifts are obtainable for $10^{12} L_{\odot}$ galaxies (corresponding to a median stellar mass of $1 \times 10^{10} \mathrm{M}_{\odot}$ ) to $z=2$ in the 300 square degree survey and to $z=4$ in the 30 square degree survey with $\sigma_{z} /(1+z) \leq 0.1$.

3. Redshifts are attainable for $10^{13} L_{\odot}$ galaxies (corresponding to stellar masses in excess of $10^{11} M_{\odot}$ ) to $z=4$ in the 300 square degree survey $\sigma_{z} /(1+z) \leq 0.05$.

4. The redshift uncertainties are a function of redshift, galaxy luminosity, map depth, and strength of the PAH features relative to the continuum.

5. In the case of very warm dust, which represents an extreme case of entirely AGNdominated galaxies, the redshifts have large uncertainties. However, even in this case, redshifts become detectable at $z>2$ for deep surveys and luminous galaxies because the dust continuum is not strong below rest-frame $10 \mu \mathrm{m}$ and there is a strong $3 \mu \mathrm{m}$ emission line, which is redshifted well into the GEP-I bands, and silicate absorption can still be present.

A comprehensive set of simulations is required to quantify redshift measurements as a function of GEP-I spectral resolving power, AGN fraction and other sources of weak PAH emission (e.g., low metallicity), galaxy-to-galaxy variability, and instrument parameters, such as nonideal bandpass shapes and $1 / f$ noise. These simulations likely represent best-case scenarios. These effects will be explored as a subsequent effort.

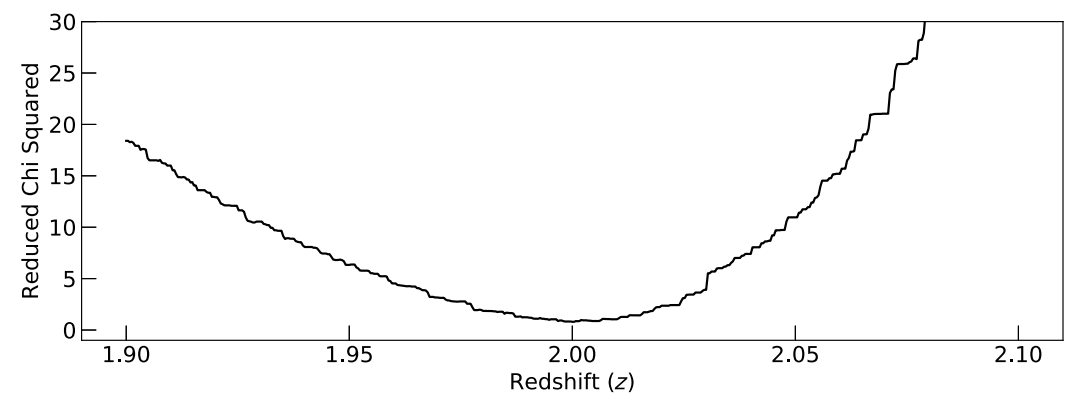

Fig. 19 The redshift reduced chi-squared distribution for a $z=2, L=10^{12} L_{\odot}$ galaxy with the model spectrum shown in Fig. 2. The depth corresponds to the 30 square degree survey. The measured redshift is $z=2 \pm 0.02$, with $\sigma_{z} /(1+z)=0.01$. This represents a best case because the observed spectrum was fit to the same model spectrum (same dust parameters) and ideal square $R=8$ bandpasses were assumed. Uncertainties from real GEP observations will be larger; however, this analysis shows that the multiple PAH emission lines combined with the deep silicate absorption can in principle lead to precise redshift determination. The jagged nature of the chi-squared distribution arises from the sampling of the model spectrum. 


\subsubsection{GEP Redshift Measurements for Galaxies with Strong AGN}

GEP has two means for measuring redshifts. One, GEP's $R=200$ spectrometer GEP-S will provide redshifts for AGN-dominated galaxies via (low- and) high-ionization fine-structure lines. Two, even in the instances of weak PAH-to-continuum emission in strongly AGNdominated galaxies, the $10-\mu \mathrm{m}$ rest-frame silicate absorption feature will still enable redshift measurements. The harsh environments immediately around AGNs can in theory destroy PAHs, ${ }^{144,145}$ but observationally, even galaxies with AGN often display these features, although they are weaker. Detailed Spitzer IRS spectra of hundreds of galaxies with AGN activity found that some PAH features are seen in most cases, ${ }^{146}$ showing that PAHs can survive in some of these environments. $\mathrm{Li}^{147}$ summarized the PAH observations in AGN by noting that the 6.2, 7.7, and $8.6 \mu \mathrm{m}$ PAH features, although suppressed with respect to the $11.3 \mu \mathrm{m}$ band (perhaps because smaller PAHs are more susceptible to the hard radiation field of an AGN) nevertheless can be present. Figure 5 in Ref. 147 of the relative PAH line strengths shows the influence of radiation hardness, as well as the limits of current modeling in Seyfert galaxies, many of which show strong neutral PAH emission.

From a theoretical perspective, coauthors of this paper have examined the role of AGN activity in a galaxy mid-IR SEDs in another paper $^{148}$ using a Gadget-2 simulation of an idealized (noncosmological) major merger of two identical disk galaxies. ${ }^{149}$ The galaxies had initial halo and baryonic masses of $9 \times 10^{12}$ and $4 \times 10^{11} M_{\odot}$, and a central black hole mass of $1.4 \times 10^{5} M_{\odot}$. The star formation and feedback were modeled as described in Refs. 150 and 149. The radiative transfer code SUNRISE ${ }^{151}$ was used to compute SEDs for seven viewing angles every $10 \mathrm{Myr}$ throughout the simulation run, and by varying the efficiency of the AGN it was effectively turned off or raised to $100 \%$ (for Eddington-limited Bondi-Hoyle accretion). The ratio of the two resultant SEDs reveals the influence of the pure AGN (see also Fig. 9 in Ref. 152). The results presented in Fig. 1 of Ref. 148 show that the silicate absorption feature is present and will enable redshift measurements even when galaxies' luminosities are strongly dominated by AGN.

Other physical effects will also influence the strengths of these mid-IR PAHs features, for example, low metallicity in high- $z$, young galaxies, or destructive supernovae activity. ${ }^{147}$ Although weak PAH features make photometric redshift measurements more challenging, it is precisely the complexity and variability of these features that ultimately enable diagnosis of the physical conditions present.

\subsubsection{Galaxy Luminosity Functions, Star Formation History, Separating AGN, and Clustering}

GEP's science objectives require luminosity functions over a range of redshifts to observe the changes in galaxy formation and the build-up of stellar mass over cosmic time. Faint-end (below $L^{*}$ ) mid- and far-infrared luminosity function slopes have not been measured above $z=0.5$, and there is disagreement about the faint-end slopes even at $z \sim 0$. GEP-I's surveys will detect hundreds of millions of galaxies and measure redshifts for millions of them for derivation of luminosity functions. With GEP-I surveys, faint-end slopes below $\log _{10}\left(L_{\mathrm{IR}} / L_{\odot}\right)=11$ for $z=0.5$, below $\log _{10}\left(L_{\mathrm{IR}} / L_{\odot}\right)=11.5$ at $z=1$, and below $\log _{10}\left(L_{\mathrm{IR}} / L_{\odot}\right)=12$ at $z=2$, will be measured. Figure 19 shows current observational determinations of the infrared bolometric luminosity function and compares these to a sample luminosity function derived from our mock GEP catalogs.

One of GEP's primary goals is to quantify the star formation history of the Universe by utilizing very large statistical samples of galaxies that overcome Poisson statistics and sample variance from large-scale structure. How well will it do? Figure 14 showed that Poisson statistics and sample variance will be overcome and Fig. 20 demonstrated that luminosity functions will be well quantified even below $L^{*}$ at $z=1$. How do these translate into measurement of the history of cosmic star formation rate density? Figure 21 shows that the star formation history will be quantified with uncertainties an order of magnitude below the state of the art to at least $z=3$. Uncertainties will likely improve substantially beyond $z=3$ also; however, that was not included in the simulations (Sec. 5.2). Because it maps large areas, GEP will also be effective at detecting gravitationally lensed galaxies. This will enable it to serendipitously detect sub- $L^{*}$ 


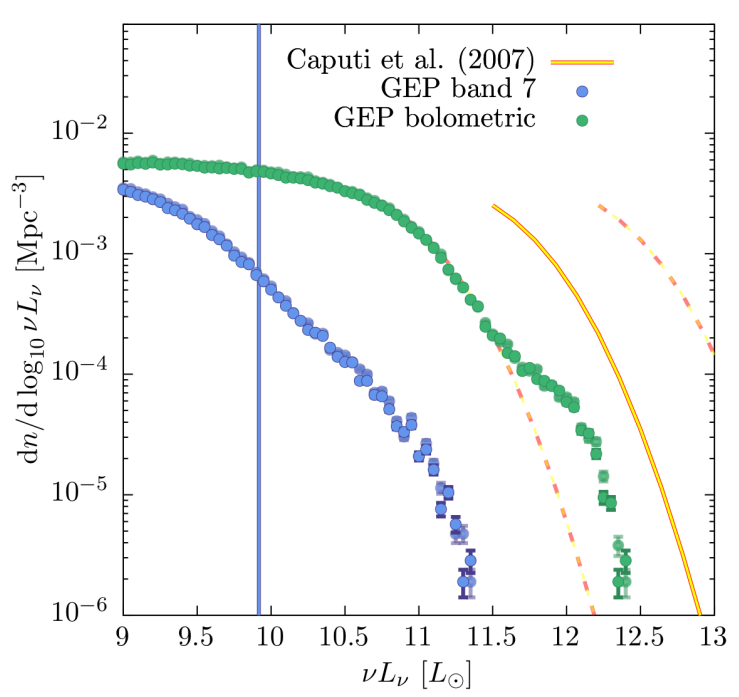

Fig. 20 GEP luminosity function for $1.0<z<1.2$ derived from the Galacticus mock catalogs assuming a $\sigma_{z} /(1+z)=0.1$ uncertainty on galaxy redshifts. Error bars are estimated assuming Poisson statistics scaled from the 4 square degree area of our mocks to 30 square degrees. Blue points show the luminosity function in the GEP-I band $7(21.2$ to $24.0 \mu \mathrm{m})$, while green points show the bolometric infrared luminosity function. The bolometric luminosity function is not shown below $10^{9} L_{\odot}$ because it becomes incomplete due to the resolution of the simulation. The blue line is the $3 \sigma$ detection limit. Faint points indicate the luminosity function that would be obtained if spectroscopic redshifts were available; it is almost indistinguishable from that constructed using photometric redshifts. The orange lines are from Ref. 25.

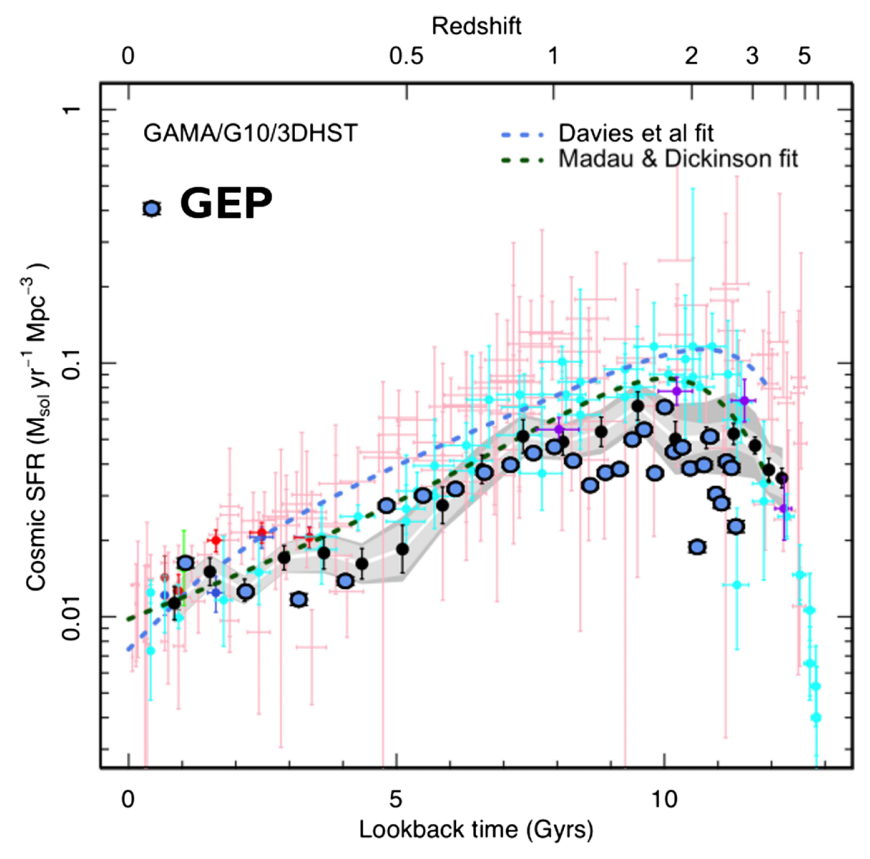

Fig. 21 A recent study of the cosmic star formation density history from deep multiband data-for which the principal source of uncertainty is the limitation of the far-infrared data-reproduced from Fig. 13 of Ref. 153 with GEP simulation data overplotted. Blue circles show expectations from a combination of GEP full sky, 300, 30, and 3 square degree surveys (for $0<z<3$ only), showing that GEP will measure the star formation history with unprecedented precision. In most cases, error bars are smaller than the symbols. The large scatter in the GEP points arises from simulations of limited cosmic volume. While the error bar sizes are appropriate for the GEP surveys, the scatter in the GEP survey data should be very small compared with what is seen here. 

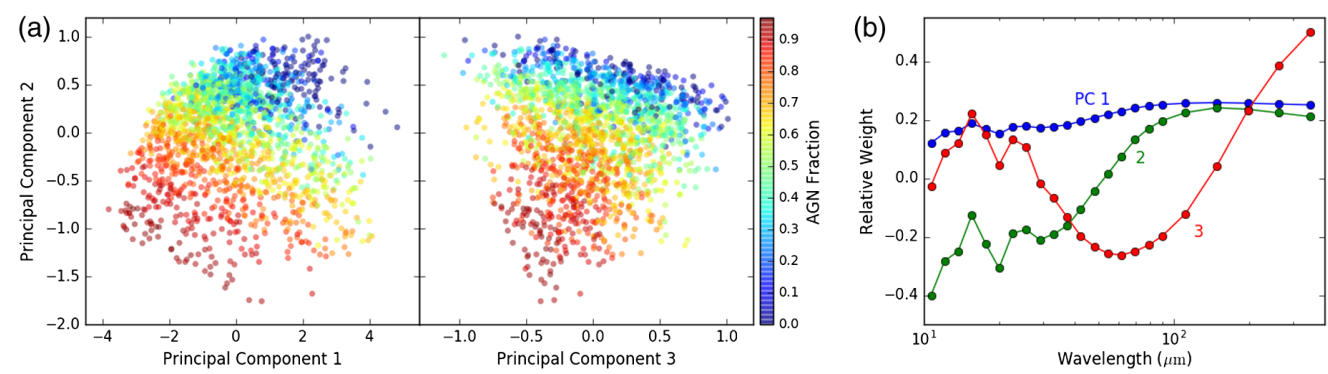

Fig. 22 GEP-I's multiband observations will enable the relative contributions of galaxy infrared luminosities from AGN and star formation to be discriminated based on their different mid-and far-infrared spectra. (a) Outcome for analysis of the 3-square degree survey at redshift $z=1.0 \pm$ 0.1 when all GEP bands are detected. Results are similar for other survey depths and subsets of GEP bands provided adequate detection rates. (b) The three principal components identified by the algorithm. Together, they measure the AGN fraction, radiation field hardness, and the redshift.

galaxies at $z=2$ with a tail out to higher redshifts, perhaps $10^{4}$ lensed galaxies in the all-sky survey and 50 in the deeper 300-square degree survey.

Another GEP goal is to identify embedded AGN and quantify their contributions to the infrared luminosities of galaxies. The mid-infrared part of the spectrum provides excellent leverage for this because the dust is hotter-thus the spectra are "bluer"- and the PAHs are more subsumed by the bright dust continuum. Figure 22 shows that a simple principal component analysis with only three components is sufficient to identify AGN and separate their contributions to infrared luminosities from star formation with GEP-I data. The high-ionization lines observed with GEP-S will indicate the presence of AGN and enable the larger catalog of GEP-I galaxies to be calibrated against the fine-structure line luminosities. These observations can be used to assess AGN influence on galactic interstellar media and the coevolution of supermassive black holes and star formation.

Within a $\Lambda C D M$ cosmology, the clustering of galaxies reveals the masses of dark matter haloes that they occupy. Galaxy mass correlates strongly with halo mass; however, it is possible that mid- and far-infrared luminosities of galaxies correlate more weakly with halo mass because even low-mass galaxies can be temporarily infrared-bright from star formation bursts. Clustering of GEP-I catalogs will strongly test galaxy formation and evolution theories that predict halo occupations as a function of redshift (Fig. 23).

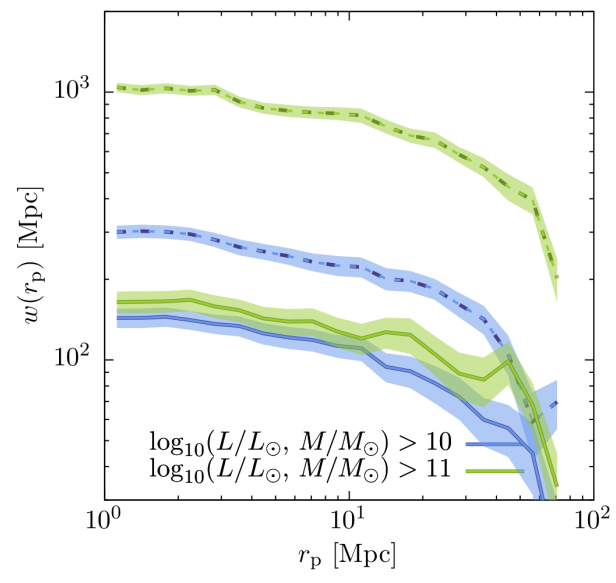

Fig. 23 Projected correlation functions from the mock catalog for galaxies selected by bolometric infrared luminosity (solid lines) and stellar mass (dashed lines) in a $1.0<z<1.2$ redshift slice. Bands indicate the expected $1 \sigma$ uncertainties for the 30 square degree survey. The Galacticus simulations show what is already known: galaxy mass correlates strongly with halo mass, leading to a strong dependence of clustering strength with mass. Conversely, however, it predicts that bolometric infrared luminosity (which indicates star-formation rate) is weakly correlated with halo mass because even galaxies in low mass halos can have occasional strong starbursts, leading to a weak dependence of clustering strength on infrared luminosity. 


\subsubsection{Milky Way and Nearby Galaxies}

GEP will map the Milky Way and the Magellanic Clouds with GEP-I during the all-sky survey. The sensitivity required of the telescope and instruments for the deep extragalactic observations will enable high signal-to-noise ratio mid- and far-infrared spectral energy distributions of starformation regions. The 3.43" mid-infrared resolution will resolve gradients in dust temperatures and PAH excitation (Fig. 24), thereby probing the radiation fields and chemistry, whereas the far-infrared spectral energy distributions will yield luminosities of embedded stars, dust temperatures, and gas masses.

\subsubsection{Interstellar Physical Conditions}

Like the multiband imager, GEP-S is designed for rapid surveys but targeting the gas-phase spectral features in the rest-frame mid- and far-infrared. The spectra from GEP-S will chart the cosmic history of the interaction of galaxies' energy sources (stars and supermassive black holes) with their gas reservoirs. Transitions from low-ionization states, such as [C II], [N II], [O III], and [NeII], can be used to infer the masses of interstellar neutral and ionized gas components, the hardness of the ultraviolet radiation fields and its implications for the stellar initial mass function,

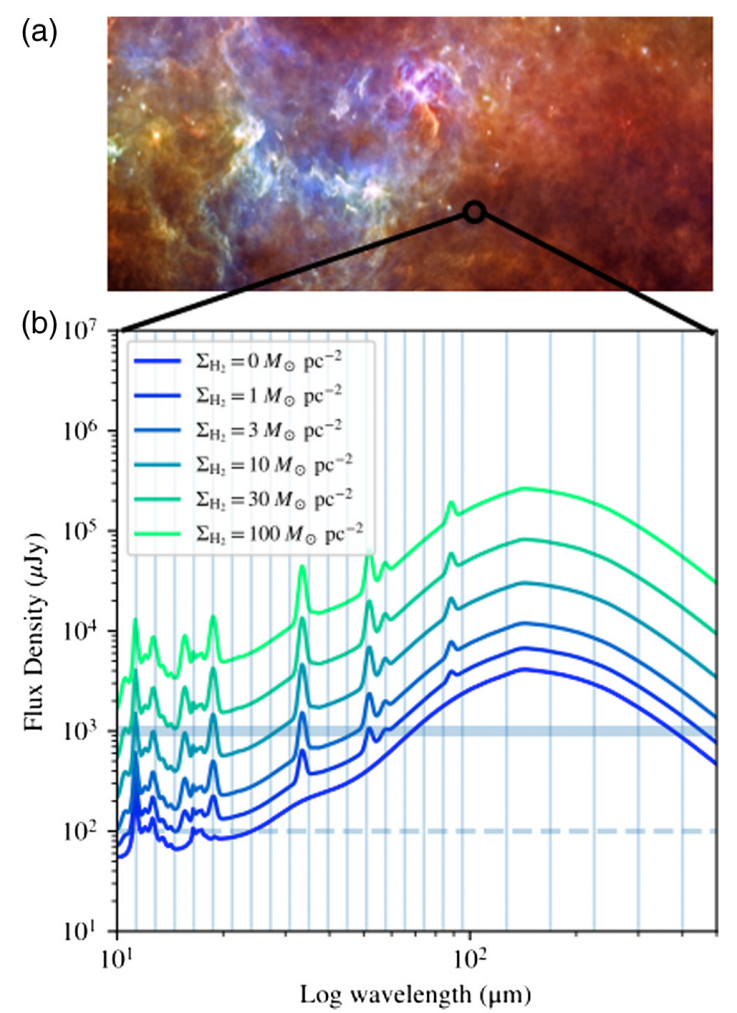

Fig. 24 GEP-I will provide spatially resolved spectral energy distribution mapping across the starforming interstellar medium over $10^{7.5}$ lines of sight in the Milky Way and nearby galaxies, providing a large data set to understand how local environment establishes interstellar conditions. The figure shows how the dust emission from (a) the Aquila molecular cloud would be mapped into (b) spatially resolved spectral energy distributions over the GEP-I bands (band edges denoted with blue vertical lines) with flux density estimates in 3 arc sec apertures. The curves correspond to the expected signatures for varying levels of molecular gas in a stellar population with a stellar surface density of $300 \mathrm{M}_{\odot} \mathrm{pc}^{-2}$. The spectral slopes, fine-structure line-to-continuum ratios, and $\mathrm{PAH}$ feature strengths vary depending on the gas surface densities. The horizontal lines indicate the approximate $5 \sigma$ depths of the all-sky survey (solid) and 300 square degree survey (dashed). The depth flux densities are lower limits because they do not account for the increased background in the galactic plane. In addition, detector nonlinearities from large backgrounds have not been modeled or accounted for. 
and the density of HII regions from which the pressure in the ISM can be inferred. Intermediate ionization transitions of nitrogen and oxygen, [NIII] and [OIII], indicated the degree of stellar processing, a proxy for metallicity ${ }^{154}$ in galaxies irrespective of the dust and gas temperature uncertainties. Finally, spectral tracers of highly ionized gas, such as [S IV] at $10.5 \mu \mathrm{m}$, [Ne V] at 14.3 and $24.3 \mu \mathrm{m}$, and [O IV] at $25.9 \mu \mathrm{m}$, with ionization potentials of 35 to $97 \mathrm{eV}$, when combined with bright lines with much lower ionization potential (e.g., [Ne II] at $12.7 \mu \mathrm{m}$ ), indicate the relative amount of heating from young stars and AGN.

To utilize this basic spectroscopic toolkit, there are three types of observations envisioned for GEP-S: (1) "blind" field-filling spectroscopic surveys obtained by rastering GEP-S on the sky, described further below; (2) individual pointed observations of specific galaxies identified in the photometric surveys or other facilities, for both precise redshifts to validate the photometric technique and fluxes in the full suite of far-infrared spectral features; and (3) spectral maps of nearby galaxies to study feedback and interstellar energy balance in detail.

Survey spectroscopy with GEP-S is a particular strength of the GEP long-slit spectrometer architecture, and the study envisions two spectral surveys: a deep survey over 1.5 square degrees and a wide spectroscopic survey over 100 square degrees. The resulting datasets will be used in at least three ways. First, they will detect $10^{4}$ galaxies directly in the far-infrared fine-structure transitions (and the continuum when binned). Second, the wide survey will be used to stack on the NGRST and/or Euclid grism sources to provide high signal-to-noise ratio average galaxy spectra in bins. Both surveys will be excellent datasets to measure the total cosmic luminosity density in the various far-infrared lines and ratios among integrated line intensities.

The synergy with the coming near-infrared grism surveys will be an especially powerful tool. Comparing the anticipated Euclid and NGRST grism depths ${ }^{155}$ with GEP depth in the 100 -square-degree survey suggests that stacks will access populations down to $19(7) \times 10^{10} L_{\odot}$, respectively, in a total sample of 60 (22) million galaxies, respectively, for Euclid and NGRST. The sensitivity is sufficient to form hundreds of independent bins in quantities, such as star formation rate, stellar mass, and metallicity. The resulting spectra will measure not only the bright fine-structure lines but also the "second-tier" transitions, such as [Ne V] (at 14 and $24 \mu \mathrm{m}$ rest frame), which provide dust-immune AGN indicators, and the quadrupole pure rotational $\mathrm{H}_{2}$ lines $(28,17,12, \ldots, \mu \mathrm{m})$ which indicate warm shocked gas. ${ }^{156}$ In considering the Euclid and Roman datasets, those sources that are very highly obscured may not yield clear redshifts with the GRISM-they of course cannot be included in the stacking analysis. These sources will, however, make excellent candidates for pointed spectroscopy with GEP-S, which will access the bright and dust immune far-IR fine-structure transitions of [OI] and [OIII]. At a redshift of $z=1$, the 1 -h GEP-S sensitivity of $6 \times 10^{-20} \mathrm{~W} \mathrm{~m}^{-2}$ is a good match to a $\sim 10^{11} L_{\odot}$ source whose less-obscured counterpart is included in the stacking analysis. While a sample of $\sim 100$ such sources will not have the full statistical power of the stacking analyses, it will nevertheless provide useful and otherwise inaccessible constraints on the properties of these obscured galaxies.

These spectral surveys will also be used for line intensity mapping, a powerful emerging technique in which the clustering of line-emitting galaxies is detected as fluctuations in a 3-D spatial-spectral dataset with redshift encoded as wavelength. The key virtue is that technique is sensitive to all forms of emission, not just the individually detected galaxies, so it offers the potential for absolute aggregate measurements irrespective of a single-source detection limit. GEP-S intensity mapping of mid- and far-infrared fine structure lines-and possibly PAHswill yield signals in autocorrelation, but it will also be powerful when cross-correlated with restframe optical and ultraviolet surveys and ground-based millimeter-wave surveys that separately probe stars and the atomic and molecular interstellar medium.

\subsubsection{Probing Feedback from Extraplanar Gas}

A hallmark feature of the cryogenic telescope and sensitive GEP-S spectrometer is excellent surface brightness sensitivity. This will provide a breakthrough capability for assessing feedback effects in local galaxies. Feedback in the form of stellar and AGN winds and supernova explosions are believed to eject gas and dust from star-forming galaxy disks, creating a reservoir of low-column-density gas in the outskirts of galaxies. This material exists below the threshold for 
star formation (hydrogen column density below 1 to $10 M_{\odot} \mathrm{cm}^{-2}$ ) and has been difficult to measure. GEP-S will be capable of detecting [CII] and [NII] in this material, even when the local density is well below the critical density for excitation. Sensitive maps of the diffuse gas can be obtained with GEP-S around low-redshift star-forming galaxies and AGN. These maps will provide a powerful test of the theories and models of stellar feedback which have evolved to have great detail but need testing by observations.

\subsection{Future Science Exploration}

GEP's science reach will be far broader than outlined here. For example, monochromatic probability-of-density-P(D)—fluctuation analyses have shown to be effective for constraining galaxy number counts into the confusion noise. ${ }^{140,141}$ Multicolor $\mathrm{P}(\mathrm{D})$ analyses could go much further: distinguishing between galaxy number count models and thereby constraining luminosity functions as a function of redshift to substantially greater depth than for analyses restricted to galaxies whose brightness are above individual detection thresholds. In nearby galaxies, mid- and far-infrared spectral energy distributions are needed for panchromatic spectral energy distribution fitting to constrain stellar populations, nebular conditions, star formation rates, and identify embedded AGN on a galaxy-by-galaxy basis. In the Milky Way, mapping of PAH emission in various environments, such as the vicinities of hot, young stars, and photodissociation regions, will probe the radiation fields and $\mathrm{PAH}$ production, excitation, and destruction. Lastly, with the possibility of linear-variable filters with $R>8$, the optimum resolution for PAH redshifts should be revisited. Then, GEP-I redshift precision should be quantified as a function of galaxy luminosity, redshift, AGN fraction (with strong AGN mid-infrared continuum leading to weak PAH lines), and instrumental parameters, such as spectral resolving power $R$.

Finally, it must be noted that the GEP design reference mission is designed for dedicated surveys. The surveys should be designed with community input and consideration of the rich landscape of imminent multiwavelength galaxy surveys. And, while the design reference mission does not support an open-time phase to manage costs, with no expendable cryogens the expected lifetime of the mission exceeds the planned survey durations. An extended mission with guest-observer opportunities would yield a large volume of science, as the Spitzer extended mission has shown.

\section{Conclusions}

The GEP concept was developed to make precise measurements of the star-formation rates, nuclear accretion rates, and interstellar conditions of galaxies over cosmic time and over the full range of cosmic environments. It was designed as a survey mission to obtain large, welldefined samples of galaxies that will not be limited by counting statistics nor by cosmic sample variance. Its science goals will be realized with $2.0 \mathrm{~m}$, cryogenic telescope $(\leq 6 \mathrm{~K})$ and two instrument modules, each with arrays of $\sim 25,000$ KIDs. It will utilize established cryogenic, telescope, and bus technologies. GEP will improve the measurement uncertainties of the cosmic star formation rate density by more than an order of magnitude out to a redshift of at least $z=3$, the current extent of our predictive simulations. Obscured AGN in galactic centers will be identified on a galaxy-by-galaxy basis, yielding new luminosity functions. Critical measurements of metallicities and interstellar conditions will be obtained for galaxies, complementary to probes at other wavelengths. GEP's ability to provide these powerful new observational capabilities derives from emerging mid- and far-infrared detector technologies that require continued development and nurturing to realize not only GEP but also a future flagship opportunity, such as OST.

\section{Acknowledgments}

This work was supported, in part, by a NASA Astrophysics Probe Concept Study grant to Jason Glenn (NASA Award Number NNX17AJ89G). This research was funded in part by the Jet Propulsion Laboratory, California Institute of Technology, under a contract with the NASA. 
Jason Glenn thanks JPL and Ball Aerospace for their strong support of the study effort. The GEP team would like to thank many contributors whose important work helped the successful completion of this study but whose roles traditionally do not qualify for inclusion in authorship lists of scientific publications: engineering aides, documentarians, administrators, administrative aides, and reviewers. The authors have no relevant financial interests in the manuscript and no other potential conflicts of interest to disclose.

\section{References}

1. F. Shankar, D. H. Weinberg, and J. Miralda-Escudé, "Self-consistent models of the AGN and black hole populations: duty cycles, accretion rates, and the mean radiative efficiency," Astrophys. J. 690, 20-41 (2009).

2. P. Madau and M. Dickinson, "Cosmic star-formation history," Annu. Rev. Astron. Astrophys. 52, 415-486 (2014).

3. J. S. Speagle et al., "A highly consistent framework for the evolution of the star-forming "main sequence" from $z \sim 0-6, "$ Astro. Phys. J. Suppl. Ser. 214, 15 (2014).

4. L. A. M. Tasca et al., "The evolving star formation rate: $M^{*}$ relation and sSFR since $z \simeq 5$ from the VUDS spectroscopic survey," Astron. Astrophys. 581, A54 (2015).

5. A. Dekel and Y. Birnboim, "Galaxy bimodality due to cold flows and shock heating," Mon. Not. R. Astron. Soc. 368(1), 2-20 (2006).

6. M. R. Blanton and J. Moustakas, "Physical properties and environments of nearby galaxies," Ann. Rev. Astron. Astrophys. 47, 159-210 (2009).

7. P. S. Behroozi, R. H. Wechsler, and C. Conroy, "The average star formation histories of galaxies in dark matter halos from $\mathrm{z}=0-8$," Astrophys. J. 770, 57 (2013).

8. J. Schaye et al., "The EAGLE project: simulating the evolution and assembly of galaxies and their environments," Mon. Not. R. Astron. Soc. 446, 521-554 (2015).

9. A. C. Fabian, "Observational evidence of active galactic nuclei feedback," Annu. Rev. 50, 455-489 (2012).

10. D. J. Croton et al., "The many lives of active galactic nuclei: cooling flows, black holes and the luminosities and colours of galaxies," Mon. Not. R. Astron. Soc. 365(1), 11-28 (2006).

11. D. Farrah et al., "Direct evidence for termination of obscured star formation by radiatively driven outflows in reddened QSOs," Astrophys. J. 745, 178 (2012).

12. J. Silk, "Unleashing positive feedback: linking the rates of star formation, supermassive black hole accretion, and outflows in distant galaxies," Astrophys. J. 772(2), 112 (2013).

13. C. Cicone et al., "Massive molecular outflows and evidence for AGN feedback from CO observations," Astron. Astrophys. 562, A21 (2014).

14. G. Neugebauer et al., "The infrared astronomical satellite (IRAS) mission," Astrophys. J. 278, L1-L6 (1984).

15. M. F. Kessler et al., "The infrared space observatory (ISO) mission," Astron. Astrophys. 315, L27-L31 (1996).

16. H. Murakami et al., "The infrared astronomical mission AKARI*," Publ. Astron. Soc. Jpn. 59, S369-S376 (2007).

17. G. L. Pilbratt et al., "Herschel space observatory: an ESA facility for far-infrared and submillimetre astronomy," Astron. Astrophys. 518, L1 (2010).

18. E. L. Wright et al., "The wide-field infrared survey explorer (WISE): mission description and initial on-orbit performance," Astron. J. 140, 1868-1881 (2010).

19. M. W. Werner et al., "The Spitzer Space Telescope mission,” Astrophys. J. Suppl. Ser. 154, 1-9 (2004).

20. D. Farrah et al., "Far-infrared instrumentation and technological development for the next decade," J. Astron. Telesc. Instrum. Syst. 5(2), 020901 (2019).

21. J. Glenn et al., "Galaxy Evolution Probe," NASA Concept Study Report, https://smd-prod .s3.amazonaws.com/science-red/s3fs-public/atoms/files/GEP_Study_Rpt.pdf (2019).

22. J. Glenn et al., "The Galaxy Evolution Probe," Astro 2020 Decadal Survey Project white paper, http://surveygizmoresponseuploads.s3.amazonaws.com/fileuploads/623127/ 5043187/183-0ec368750b918c40030f7019cf8bbaea_GlennJason.pdf (2019). 
23. J. Glenn et al., "The Galaxy Evolution Probe: a concept for a mid and far-infrared space observatory," Proc. SPIE 10698, 106980L (2018).

24. B. Moore et al., "Thermal architecture of the galaxy evolution probe mission concept," Proc. SPIE 10698, 1069858 (2018).

25. K. I. Caputi et al., "The infrared luminosity function of galaxies at redshifts $z=1$ and $z \sim 2$ in the GOODS fields," Astrophys. J. 660, 97-116 (2007).

26. R. C. Kennicutt, Jr., "The global Schmidt law in star-forming galaxies," Astrophys. J. 498(2), 541 (1998).

27. A. J. Battisti et al., "Continuous mid-infrared star formation rate indicators: diagnostics for $0<z<3$ star-forming galaxies," Astrophys. J. 800, 143 (2015).

28. D. A. Dale et al., "A two-parameter model for the infrared/submillimeter/radio spectral energy distributions of galaxies and active galactic nuclei," Astrophys. J. 784(1), 83 (2014).

29. H. Teplitz et al., "Measuring PAH emission in ultradeep Spitzer IRS spectroscopy of high-redshift IR-luminous galaxies," Astrophys. J. 659(2), 941 (2007).

30. D. A. Riechers et al., "Polycyclic aromatic hydrocarbon and mid-infrared continuum emission in a $z>4$ submillimeter galaxy," Astrophys. J. 786(1), 31 (2014).

31. I. De Looze et al., "The applicability of far-infrared fine-structure lines as star formation rate tracers over wide ranges of metallicities and galaxy types," Astron. Astrophys. 568, A62 (2014).

32. S. Mordini, L. Spinoglio, and J. A. Fernández-Ontiveros, "Calibration of mid- to farinfrared spectral lines in galaxies," arXiv:2105.04584 (2021).

33. J. Magorrian et al., "The demography of massive dark objects in galaxy centers," Astron. J. 115(6), 2285 (1998).

34. A. Marconi and L. K. Hunt, "The relation between black hole mass, bulge mass, and nearinfrared luminosity," Astrophys. J. Lett. 589(1), L21 (2003).

35. J. Silk and M. Rees, "Quasars and galaxy formation," Astron. Astrophys. 331, L1-L4 (1998).

36. R. Bower et al., "Breaking the hierarchy of galaxy formation," Mon. Not. R. Astron. Soc. 370(2), 645-655 (2006).

37. D. Sijacki et al., "A unified model for AGN feedback in cosmological simulations of structure formation," Mon. Not. R. Astron. Soc. 380(3), 877-900 (2007).

38. T. Di Matteo et al., "Direct cosmological simulations of the growth of black holes and galaxies," Astrophys. J. 676(1), 33 (2008).

39. A. Benson et al., "What shapes the luminosity function of galaxies?" Astrophys. J. 599(1), 38 (2003).

40. A. Wagner et al., "Galaxy-scale AGN feedback-theory," Astron. Nachr. 337(1-2), 167-174 (2016).

41. A. Vayner et al., "Powerful winds in high-redshift obscured and red quasars," Mon. Not. R. Astron. Soc. 504, 4445-4459 (2021).

42. M. Pereira-Santaella et al., "Far-infrared metallicity diagnostics: application to local ultraluminous infrared galaxies," Mon. Not. R. Astron. Soc. 470, 1218-1232 (2017).

43. J. A. Fernández-Ontiveros et al., "Measuring chemical abundances with infrared nebular lines: HII-Chi-mistry-IR," in Astron. Astrophys. 652, 23 (2021).

44. P. K. Day et al., "A broadband superconducting detector suitable for use in large arrays," Nature 425(6960), 817-821 (2003).

45. J. J. Baselmans et al., "A kilo-pixel imaging system for future space based far-infrared observatories using microwave kinetic inductance detectors," Astron. Astrophys. 601, A89 (2017).

46. S. Hailey-Dunsheath et al., "Development of aluminum LEKIDs for balloon-borne far-IR spectroscopy," J. Low Temp. Phys. 193(5-6), 968-975 (2018).

47. N. P. Lourie et al., "Preflight characterization of the BLAST-TNG receiver and detector arrays," Proc. SPIE 10708, 107080L (2018).

48. T. Nikola et al., "HIRMES: the third generation instrument for SOFIA (conference presentation)," Proc. SPIE 10708, 1070800 (2018).

49. C. M. McKenney et al., "Design considerations for a background limited 350 micron pixel array using lumped element superconducting microresonators," Proc. SPIE 8452, 84520S (2012). 
50. L. J. Swenson et al., "MAKO: a pathfinder instrument for on-sky demonstration of low-cost 350 micron imaging arrays," Proc. SPIE 8452, 84520P (2012).

51. R. Adam et al., "The NIKA2 large-field-of-view millimetre continuum camera for the 30 m IRAM telescope," Astron. Astrophys. 609, A115 (2018).

52. A. Paiella et al., "Kinetic inductance detectors for the OLIMPO experiment: design and pre-flight characterization," J. Cosmol. Astropart. Phys. 2019(01), 039 (2019).

53. C. M. Bradford et al., "The origins survey spectrometer (OSS): a far-IR discovery machine for the Origins Space Telescope," Proc. SPIE 10698, 1069818 (2018).

54. A. Poglitsch et al., "The photodetector array camera and spectrometer (PACS) on the Herschel space observatory," Astron. Astrophys. 518, L2 (2010).

55. R. Hijmering et al., "Readout of a 176 pixel FDM system for SAFARI TES arrays," Proc. SPIE 9914, 99141C (2016).

56. G. de Lange et al., "The SAFARI grating spectrometer for the SPICA space observatory (conference presentation)," Proc. SPIE 10708, 107081N (2018).

57. W. Holland et al., "Scuba-2: the 10000 pixel bolometer camera on the James Clerk Maxwell telescope," Mon. Not. R. Astron. Soc. 430(4), 2513-2533 (2013).

58. M. J. Griffin et al., "The Herschel-Spire instrument and its in-flight performance," Astron. Astrophys. 518, L3 (2010).

59. J. D. Wheeler et al., "Superspec: the on-chip spectrometer: characterization of a full 300 channel filterbank (conference presentation)," Proc. SPIE 10708, 107080P (2018).

60. A. Barlis et al., "Development of aluminum LEKIDS for ballooon-borne far-infrared spectroscopy (conference presentation)," Proc. SPIE 10708, 107080V (2018).

61. J. Aguirre et al., "STARFIRE: the spectroscopic terahertz airborne receiver for far-infrared exploration," AAS 231, 32804 (2018).

62. G. W. Wilson et al., "The TolTEC project: a millimeter wavelength imaging polarimeter (conference presentation)," Proc. SPIE 10708, 107080I (2018).

63. J. Perido et al., "Extending KIDs to the mid-IR for future space and suborbital observatories," J. Low Temp. Phys. 199, 696-703 (2020).

64. F. Defrance et al., "16:1 bandwidth two-layer antireflection structure for silicon matched to the 190-310 GHz atmospheric window," Appl. Opt. 57, 5196 (2018).

65. P. Savander, "Microlens arrays etched in glass and silicon," Opt. Lasers Eng. 20, 97-107 (1994).

66. K. P. Larsen, J. T. Ravnkilde, and O. Hansen, "Investigations of the isotropic etch of an ICP source for silicon microlens mold fabrication," J. Micromech. Microeng. 15, 873-882 (2005).

67. C.-F. Chen et al., "Silicon microlens structures fabricated by scanning-probe gray-scale oxidation," Opt. Lett. 30, 652-654 (2005).

68. P. N. A. Belmonte et al., "Microfabrication and characterization of single-mask silicon microlens arrays for the IR spectra," Proc. SPIE 9130, 91300D (2014).

69. Z. Deng et al., "Fabrication of large-area concave microlens array on silicon by femtosecond laser micromachining," Opt. Lett. 40, 1928-1931 (2015).

70. X. Meng et al., "Simple fabrication of closed-packed IR microlens arrays on silicon by femtosecond laser wet etching," Appl. Phys. A 121, 157-162 (2015).

71. H. Zuo et al., "CMOS compatible fabrication of micro, nano convex silicon lens arrays by conformal chemical vapor deposition," Opt. Express 25, 3069-3076 (2017).

72. J. F. Gonzàlez et al., "Infrared antennas coupled to lithographic Fresnel zone plate lenses," Appl. Opt. 43, 6067-6073 (2004).

73. B. A. Mazin et al., "Digital readouts for large microwave low-temperature detector arrays," Nucl. Instrum. Methods Phys. Res. Sect. A 559(2), 799-801 (2006).

74. S. Yates et al., "Fast Fourier transform spectrometer readout for large arrays of microwave kinetic inductance detectors," Appl. Phys. Lett. 95(4), 042504 (2009).

75. R. Duan et al., "An open-source readout for MKIDs," Proc. SPIE 7741, 77411V (2010).

76. R. Duan, "Instrumentation for kinetic-inductance-detector-based submillimeter radio astronomy," PhD thesis, California Institute of Technology (2015).

77. S. McHugh et al., "A readout for large arrays of microwave kinetic inductance detectors," Rev. Sci. Instrum. 83(4), 044702 (2012). 
78. M. J. Strader, "Digital readout for microwave kinetic inductance detectors and applications in high time resolution astronomy," PhD thesis, UC Santa Barbara (2016).

79. O. Bourrion et al., "NIKEL_AMC: readout electronics for the NIKA2 experiment," J. Instrum. 11(11), P11001 (2016).

80. J. van Rantwijk et al., "Multiplexed readout for 1000-pixel arrays of microwave kinetic inductance detectors," IEEE Trans. Microwave Theory Tech. 64(6), 1876-1883 (2016).

81. S. Gordon et al., "An open source, FPGA-based LEKID readout for blast-TNG: pre-flight results," J. Astron. Instrum. 5(04), 1641003 (2016).

82. S. W. Henderson et al., "Highly-multiplexed microwave SQUID readout using the SLAC microresonator radio frequency (SMuRF) electronics for future CMB and sub-millimeter surveys," Proc. SPIE 10708, 1070819 (2018).

83. T. Müller et al., "Herschel celestial calibration sources," Exp. Astron. 37(2), 253-330 (2014).

84. O. Noroozian et al., "Crosstalk reduction for superconducting microwave resonator arrays," IEEE Trans. Microwave Theory Tech. 60(5), 1235-1243 (2012).

85. S. J. Yates et al., "Surface wave control for large arrays of microwave kinetic inductance detectors," IEEE Trans. Terahertz Sci. Technol. 7(6), 789-799 (2017).

86. X. Liu et al., "Superconducting micro-resonator arrays with ideal frequency spacing," Appl. Phys. Lett. 111(25), 252601 (2017).

87. S. Shu et al., "Increased multiplexing of superconducting microresonator arrays by postcharacterization adaptation of the on-chip capacitors," Appl. Phys. Lett. 113(8), 082603 (2018).

88. S. Trimberger, "Three ages of FPGAs: a retrospective on the first thirty years of FPGA technology," Proc. IEEE 103, 318-331 (2015).

89. Collaboration for Astronomy Signal Processing and Electronics Research, "ROACH-1, (reconfigurable open architecture computing hardware) standalone FPGA processing board," 2008, https://casper.ssl.berkeley.edu/wiki/ROACH.

90. P. De Bernardis et al., "Exploring cosmic origins with core: the instrument," J. Cosmol. Astropart. Phys. 2018(04), 015 (2018).

91. D. Elftmann, "Xilinx on-orbit reconfigurable kintex ultrascale FPGA technology for space," in SEFUW: SpacE FPGA Users Workshop, 4th Ed., Eur. Space Res. and Technol. Centre (ESTEC) (2018).

92. Xilinx, "Virtex-6 family overview," DS150 v2.5 (2015).

93. Xilinx, "Ultrascale FPGA product tables and product selection guide," https://www.xilinx .com/support/documentation/selection-guides/ultrascale-fpga-product-selection-guide.pdf (2013-2016).

94. Xilinx, "Virtex-5qv family overview," DS192 v1.6 (2018).

95. Xilinx, "Zynq ultrascale+ RFSOC overview," DS889 v1.9 (2019).

96. Xilinx, "RT kintex ultrascale FPGAS for ultra high throughput and high bandwidth applications," WP523 v1.0 (2020).

97. Xilinx, "Virtex-5 family overview," DS100 v5.1 (2015).

98. M. Wirthlin, "FPGAs operating in a radiation environment: lessons learned from FPGAs in space," J. Instrum. 8(02), C02020 (2013).

99. G. Swift, "Invited talk I: the foundations of robustness in reconfigurability in a radiation environment: understanding single-event effects test results on SRAM-based FPGAs," in NASA/ESA Conf. Adapt. Hardware and Syst. (AHS), IEEE, pp. xv-xv (2017).

100. L. Raczkowski, "Single event effects," in 27th Annu. Single Event Effects (SEE) Symp. Coupled with the Mil. and Aerosp. Programm. Logic Devices (MAPLD) Workshop, San Diego, California, IEEE (2018).

101. “SpacE FPGA users workshop,” SEFUW: SpacE FPGA Users Workshop, 4th Ed., https:// indico.esa.int/event/232/contributions/2147/attachments/1800/2100/SEFUW_2018_FPGA_ and_SOC_Airbus_Defence_Space.pdf (accessed 9-11 April 2018).

102. J. Le Mauff and E. Lepape, "From eFPGA cores to RHBD SoC FPGA," in 4th SpacE FPGA Users Workshop (SEFUW) (2018).

103. I. Wang et al., "LiveCheckHSI: a hardware/software co-verification tool for hyperspectral imaging systems with embedded system-on-chip instrument avionics," in IEEE Aerospace Conf., IEEE, pp. 1-11 (2018). 
104. D. S. Lee et al., "Single-event characterization of the $20 \mathrm{~nm}$ Xilinx Kintex Ultrascale fieldprogrammable gate array under heavy ion irradiation," in IEEE Radiat. Effects Data Workshop (REDW), IEEE, pp. 1-6 (2015).

105. D. S. Lee, "Commercial field-programmable gate arrays for space processing applications," Tech. report, Sandia National Lab, Albuquerque, New Mexico (2017).

106. G. A. Allen and S. Vartanian, "NEPP FPGA update FY18 v2," NASA Electronic Parts and Packaging (NEPP) Program 2018, Building 3 Auditorium, NASA Goddard Space Flight Center, Greenbelt, MD (2018).

107. Abaco Systems, "VP430 direct RF processing system," https://www.abaco.com/products/ vp430-rfsoc-board (accessed August 2021).

108. F. Hsiao et al., "A $2.2 \mathrm{GS} / \mathrm{s} 188 \mathrm{~mW}$ spectrometer processor in $65 \mathrm{~nm}$ CMOS for supporting low-power THz planetary instruments," in IEEE Custom Integr. Circuits Conf. (CICC), IEEE, pp. 1-3 (2015).

109. A. M. Koekemoer et al., "CANDELS: the cosmic assembly near-infrared deep extragalactic legacy survey-The Hubble space telescope observations, imaging data products, and mosaics," Astrophys. J. Suppl. Ser. 197, 36 (2011).

110. M. L. N. Ashby et al., "S-CANDELS: the Spitzer-cosmic assembly near-infrared deep extragalactic survey. Survey design, photometry, and deep IRAC source counts," Astrophys. J. Suppl. Ser. 218, 33 (2015).

111. D. B. Sanders et al., "S-COSMOS: the Spitzer legacy survey of the Hubble space telescope ACS 2 deg 2 COSMOS field i: survey strategy and first analysis," Astrophys. J. Suppl. Ser. 172, 86-98 (2007).

112. L. Bianchi, B. Shiao, and D. Thilker, "Revised catalog of GALEX ultraviolet sources. I. The all-sky survey: GUVcat_AIS," Astrophys. J. Suppl. Ser. 230, 24 (2017).

113. S. P. Driver et al., "GAMA: towards a physical understanding of galaxy formation," Astron. Geophys. 50, 5.12-5.19 (2009).

114. M. E. Cluver et al., "Galaxy and mass assembly (GAMA): mid-infrared properties and empirical relations from WISE," Astrophys. J. 782, 90 (2014).

115. K. Mitchell-Wynne et al., "HerMES: a statistical measurement of the redshift distribution of Herschel-SPIRE sources using the cross-correlation technique," Astrophys. J. 753, 23 (2012).

116. B. Catinella et al., "xGASS: total cold gas scaling relations and molecular-to-atomic gas ratios of galaxies in the local Universe," Mon. Not. R. Astron. Soc. 476, 875-895 (2018).

117. A. Saintonge et al., "xCOLD GASS: the complete IRAM $30 \mathrm{~m}$ legacy survey of molecular gas for galaxy evolution studies," Astrophys. J. Suppl. Ser. 233, 22 (2017).

118. J. Freundlich et al., "PHIBSS2: survey design and $\mathrm{z}=0.5-0.8$ results. Molecular gas reservoirs during the winding-down of star formation," Astron. Astrophys. 622, A105 (2019).

119. R. Pavesi et al., "The CO luminosity density at high-z (COLDz) survey: a sensitive, largearea blind search for low-j CO emission from cold gas in the early universe with the Karl G. Jansky very large array," Astrophys. J. 864, 49 (2018).

120. F. Walter et al., "ALMA spectroscopic survey in the hubble ultra deep field: survey description," Astrophys. J 833(1), 67 (2016).

121. CEERS, "The cosmic evolution early release science survey," https://ceers.github.io/index .html (accessed August 2021).

122. Euclid Consortium, "Surveys," 18 June 2016, https://www.euclid-ec.org/?page_id=2581 (accessed August 2021).

123. "Nancy grace roman space telescope reference information," https://roman.gsfc.nasa.gov/ science/Roman_Reference_Information.html.

124. K. S. Dawson et al., "The SDSS-IV extended baryon oscillation spectroscopic survey: overview and early data," Astrophys. J 151(2), 44 (2016).

125. J. BockSPHEREx Science Team, "The SPHEREx all-sky spectral survey," in American Astronomical Society Meeting Abstracts, p. 354.21 (2018).

126. P. A. Abell et al. LSST Science Collaboration, "LSST Science Book, Version 2.0," arXiv: 0912.0201 (2009).

127. V. Springel et al., "Simulations of the formation, evolution and clustering of galaxies and quasars," Nature 435, 629-636 (2005). 
128. A. J. Benson, "GALACTICUS: a semi-analytic model of galaxy formation," New Astron. 17, 175-197 (2012).

129. D. Calzetti, "Star formation rate indicators," Secular Evolution of Galaxies, p. 419 (2013).

130. M. Béthermin et al., "Spitzer deep and wide legacy mid- and far-infrared number counts and lower limits of cosmic infrared background," Astron. Astrophys. 512, A78 (2010).

131. B. Magnelli et al., "The deepest Herschel-Pacs far-infrared survey: number counts and infrared luminosity functions from combined PEP/GOODS-H observations," Astron. Astrophys. 553, A132 (2013).

132. A. J. Benson et al., "What shapes the luminosity function of galaxies?" Astrophys. J. 599, 38-49 (2003).

133. C. Papovich et al., "Spitzer mid- to far-infrared flux densities of distant galaxies," Astrophys. J. 668, 45-61 (2007).

134. M. Bonato et al., "Origins Space Telescope: predictions for far-IR spectroscopic surveys," Publ. Astron. Soc. Aust. 36, e017 (2019).

135. "MIPS instrument handbook," MIPS Heritage Documentation, https://irsa.ipac.caltech .edu/data/SPITZER/docs/mips/mipsinstrumenthandbook (2011).

136. D. T. Frayer et al., "Spitzer 70 and $160 \mu \mathrm{m}$ observations of the cosmos field," Astron. J. 138(5), 1261 (2009).

137. S. Berta et al., "Building the cosmic infrared background brick by brick with Herschel/ Pep," Astron. Astrophys. 532, A49 (2011).

138. Herschel, "Herschel observers' manual," HERSCHEL-HSC-DOC-0876 Version 5.0.3 (2014).

139. M. Béthermin et al., "A unified empirical model for infrared galaxy counts based on the observed physical evolution of distant galaxies," Astrophys. J. Lett. 757(2), L23 (2012).

140. T. T. Takeuchi and T. T. Ishii, "A general formulation of the source confusion statistics and application to infrared galaxy surveys," Astrophys. J. 604, 40-62 (2004).

141. J. Glenn et al., "HerMES: deep galaxy number counts from a P (D) fluctuation analysis of spire science demonstration phase observations," Mon. Not. R. Astron. Soc. 409(1), 109-121 (2010).

142. I. Labbé et al., "Ultradeep IRAC imaging over the HUDF and goods-south: survey design and imaging data release," Astrophys. J. Suppl. Ser. 221(2), 23 (2015).

143. P. D. Hurley et al., "HELP: XID+, the probabilistic de-blender for Herschel SPIRE maps," Mon. Not. R. Astron. Soc. 464, 885-896 (2017).

144. P. F. Roche et al., "An atlas of mid-infrared spectra of galaxy nuclei," Mon. Not. R. Astron. Soc. 248, 606 (1991).

145. G. M. Voit, "Destruction and survival of polycyclic aromatic hydrocarbons in active galaxies," Mon. Not. R. Astron. Soc. 258, 841 (1992).

146. S. Tommasin et al., "Spitzer-IRS high-resolution spectroscopy of the $12 \mu \mathrm{m}$ Seyfert Galaxies. II. Results for the complete data set," Astrophys. J. 709, 1257-1283 (2010).

147. A. Li, "Spitzer's perspective of polycyclic aromatic hydrocarbons in galaxies," Nat. Astron. 4, 339-351 (2020).

148. J. McKinney et al., "Dust-enshrouded AGN can dominate host-galaxy cold-dust emission," Astrophys. J. (submitted).

149. V. Springel, "The cosmological simulation code GADGET-2," Mon. Not. R. Astron. Soc. 364, 1105-1134 (2005).

150. V. Springel and L. Hernquist, "Cosmological smoothed particle hydrodynamics simulations: a hybrid multiphase model for star formation," Mon. Not. R. Astron. Soc. 339, 289-311 (2003).

151. P. Jonsson, "SUNRISE: polychromatic dust radiative transfer in arbitrary geometries," Mon. Not. R. Astron. Soc. 372, 2-20 (2006).

152. J. Dietrich et al., "The AGN luminosity fraction in merging galaxies," Mon. Not. R. Astron. Soc. 480, 3562-3583 (2018).

153. S. P. Driver et al., "GAMA/G10-COSMOS/3D-HST: the $0<z<5$ cosmic star formation history, stellar-mass, and dust-mass densities," Mon. Not. R. Astron. Soc. 475(3), 2891-2935 (2018). 
154. T. Nagao, R. Maiolino, and H. Matsuhara, "Next generation study of the cosmic metallicity evolution with SPICA," in SPICA Joint Eur./Jpn. Workshop, EDP Sciences, p. 04003 (2009).

155. A. Merson et al., "Predicting $\mathrm{H} \alpha$ emission-line galaxy counts for future galaxy redshift surveys," Mon. Not. R. Astron. Soc. 474(1), 177-196 (2018).

156. P. M. Ogle, L. Lanz, and P. N. Appleton, "Jet-shocked $\mathrm{H}_{2}$ and $\mathrm{CO}$ in the anomalous arms of molecular hydrogen emission galaxy NGC 4258," Astrophys. J. Lett. 788, L33 (2014).

Jason Glenn received his BS degree in physics from the University of New Mexico in 1991 and his PhD in astronomy from the University of Arizona in 1997. Since 2020, he has been a research astrophysicist, stellar, galactic, and extragalactic in the Observational Cosmology Laboratory at the NASA Goddard Space Flight Research Center. Prior to that, he was a professor of astrophysics at the University of Colorado Boulder for 20 years. He is the author of more than 200 journal papers. His current research interests include galaxy evolution, the cool interstellar medium in galaxies, and infrared through millimeter-wave instrument and detector development.

Charles M. Bradford received his doctorate from Cornell in 2001. He held a Millikan postdoctoral fellowship at Caltech from 2001 to 2003 and has been on the science staff at Jet Propulsion Laboratory (JPL) since that time. He enjoys developing and fielding new submillimeter- and millimeter-wave instrumentation, and using spectroscopy to study interstellar medium conditions in galaxies. His current projects include a balloon-borne far-IR spectrometer, an on-chip $\mathrm{mm}$-wave spectrometer, and ultra-sensitive far-IR detectors for cryogenic space missions.

Rashied Amini, PhD, is a system engineer at JPL, California Institute of Technology, working in mission formulation and in research of autonomous technologies. Recently, he was the HabEx and Galaxy Evolution Probe Study Lead, submitted to the 2020 Astrophysics Decadal Survey. As a result of his formulation work, he is interested in supporting the maturation of technologies critical to science exploration. He received his $\mathrm{PhD}$ in physics from Washington University in St. Louis.

Lee Armus is a senior staff scientist at IPAC with over 25 years of experience in infrared spectroscopy and imaging. He is currently the lead scientist at the Roman Space Telescope Science Support Center, and formerly the lead for the IRS Instrument Support Team at the Spitzer Science Center. His research is focused on several topics central to the science of the Galaxy Evolution Probe, including galaxy mergers, galactic outflows, and luminous infrared galaxies.

Andrew J. Benson is a staff scientist at the Observatories of the Carnegie Institution for Science. His research focuses on understanding the nature of dark matter and the process of galaxy formation, with a particular emphasis on formulating a coherent picture of the many different aspects of these problems. He has developed a model of dark matter and galaxy formation physics, Galacticus, which blends both analytic understanding and numerical techniques.

Jeremy Darling is a professor at the University of Colorado. He studies osmology, galaxy evolution, massive black holes, and astrophysical approaches to fundamental physics.

Jeanette L. Domber is a program manager for Ball Aerospace Civil Space. She received her $\mathrm{PhD}$ in aerospace engineering from the University of Colorado at Boulder.

Sarah J. Lipscy is the deputy director of Civil Space Business Development at Ball Aerospace. She holds a PhD in astronomy and physics from UCLA.

Raphael Shirley is a postdoctoral researcher at the University of Southampton. His research is focused on galaxy evolution and the role of active galactic nuclei in star formation over cosmic time. He has worked on Herschel imaging, multi-wavelength astronomy, and supernova observations. He is now working on image processing pipelines for the Vera C. Rubin Observatory and the VISTA telescope. He has previously held positions at Sussex, Cambridge, and the IAC in Tenerife. 
Howard A. Smith is a senior astrophysicist at the Center for Astrophysics, Harvard \& Smithsonian (CfA) in Cambridge, with over 350 published scientific articles, and is a member of the Harvard Astronomy Department. His research field is the origins of stars in the Milky Way and other galaxies, with specialization in techniques of infrared spectroscopy and instrument development.

Jonas Zmuidzinas (BS Caltech 1981, PhD Berkeley 1987) joined the Caltech physics faculty in 1989 and currently serves as the Merle Kingsley Professor of Physics and Director of the Caltech Optical Observatories. He has also concurrently held positions at JPL as a senior research scientist (2006 to 2016), director of the JPL Microdevices Laboratory (2007 to 2011), and JPL chief technologist (2011 to 2016). His research focuses on superconducting detectors and devices and their application to astronomy.

Biographies of the other authors are not available. 